\title{
Geochemistry and Petrology of Basic Volcanic Rocks of Jabal Al Haruj Al-Aswad, Libya
}

\author{
N. M. Al-Hafdh ${ }^{*}$, Abd El-Salam S. El-Shaafi ${ }^{2}$ \\ ${ }^{1}$ Department of Earth Science, Faculty of Sciences, Mosul University, Mosul, Iraq \\ ${ }^{2}$ Department of Earth Science, Faculty of Sciences, Benghazi University, Benghazi, Libya \\ Email: *Alhafdh@yahoo.co.uk
}

Received 3 January 2015; accepted 26 January 2015; published 30 January 2015

Copyright (C) 2015 by authors and Scientific Research Publishing Inc.

This work is licensed under the Creative Commons Attribution International License (CC BY). http://creativecommons.org/licenses/by/4.0/

\section{Open Access}

\section{Abstract}

The Al Haruj Intra-continental Volcanic Province is the largest part of the extensive volcanic activity in Libya which is considered to be a typical within plate basalts. The volcano-tectonics evolution of this province, as well as its origin, are still widely disputed. According to K-Ar dating previously studied, the volcanic activity started in the Late Miocene and lasted until at least the Late Pleistocene. The field may still be volcanically active. The mafic rocks of Jabal Al Haruj have been classified into six major phases or groups. These phases have been differentiated using Landsat images together with aerial photographs of different scales as well as field observations. The topographic forms of the earliest phase are highly eroded while the forms of the latest phase are usually fresh and very well preserved as regards primary features. Mafic lavas of this field consist of alkali basalts to olivine tholeiites (transitional basalt) which contain olivine as essential constituent together with clinopyroxene, plagioclase and glass. The basalt exhibits intergranular, intersertal, ophitic and subophitic relations. Amygdaloidal and glomerporphyritic textures are also observed. The basaltic rocks of different ages and from different localities are petrographically rather similar. Phenocrysts of olivine probably the result from slow cooling in crustal magma chambers prior to eruptions, suggesting that magmas ascended slowly through the crust. 109 samples have been carefully collected from various phases, some of these samples have been chosen for major and trace elements analyses, using XRF in order to determine the characteristics of the mantle source and investigate crustal interaction. The major and trace elements revealed a slightly significant chemical diversity among the phases and within each phase. The normative classification of most of these rocks shows close agreement with their modal classification. A vague correlation between MgO and most major oxides in the studied samples suggests different degrees of partial melting rather than fractional crystallization. A characteristic feature of the studied volcanic rocks is the relatively constant ratios of certain incompatible trace elements $(\mathrm{Nb} / \mathrm{Zr}, \mathrm{Rb} / \mathrm{Zr})$,

*Corresponding author. 
which provides strong evidence of a common source. In addition, the rocks display similar patterns of the peaks and troughs; this strongly suggests that they have a common parent and common subsequent processes. The compatible transitional metals Ni (81-193 ppm) and Cr contents $(238-361 \mathrm{ppm})$ and relatively low $\mathrm{Mg \#}(\mathrm{Mg} /(\mathrm{Mg}+\mathrm{Fet}))(52-62)$ give an indication that the studied basaltic rocks have slightly to moderately fractionated olivine and/or spinel. The magmatism of this volcanic field seems to be related to reactivation of pre-existing structures during the passive rifting of the Sirt Basin that most likely produced in response to convergence between European and African plates since Jurassic until Holocene times.

\section{Keywords}

Jabal Al Haruj Al Aswad Area, Basaltic Rocks, Geochemistry, Petrology, Petrogenesis, Libya

\section{Introduction}

The Al Haruj (Jabal Al Haruj al Aswad and al Abiyad) area is located in central part of Libya, and is considered one of the main occurrences of young extrusive rocks. It is the largest of four volcanic provinces in Libya; the other three occurrences are in Gharyan province in north-western Tripoli, Jabal as Sawda between Gharyan and Jabal Al Haruj, and Jabal Nuqy farther south, close to the Tibesti massif at the Chadian-Libyan border (Figure 1).

All these occurrences represent the main Tertiary-Recent volcanics in Libya, and are arranged with older volcanics in the NNW to the youngest in the SSE. Moreover, the smallest sizes of these volcanics are encountered in the extreme south-east of Libya, in Jabal Awayant, Busrewil and Wadsworth [1]. In general, these volcanics are composed essentially of voluminous alkaline basalts and less extensive transitional and theoliitic basalts; more differentiated rocks are less common, Busrewil and Suwesi [2].

Al Haruj al Abiyad falls within the Al Haruj basalt field; it is located south-west of the main body and is the youngest region of the field, which is called Al Haruj Aswad. The extensive lava plateaus cover an area of at least 16,000 $\mathrm{km}^{2}$ in $\mathrm{Al}$ Haruj al Aswad, preserved as variably eroded lava fields. The entire Al Haruj has a volcanic area of $45,000 \mathrm{~km}^{2}$; thus, it is significantly larger than the Tibesti volcanic region, Nemeth et al. [3]. Most of the volume of the lava involved in the building of the lava plateau seems to have been extruded in an extremely fluid condition through thousands of fissures, which are not visible in the field, Busrewil and Wadsworth [4].

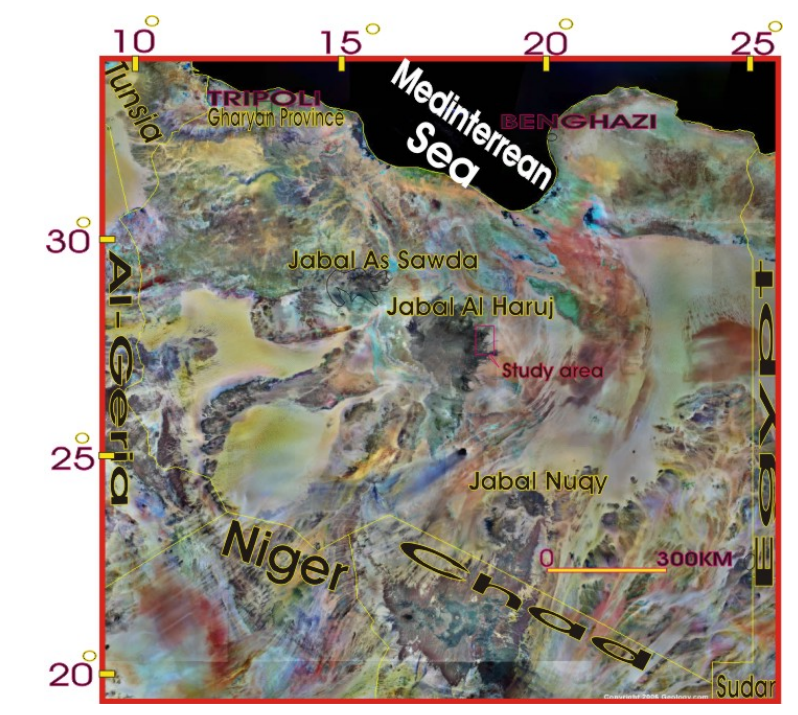

Figure 1. Landsat image showing the main occurrences of Tertiary volcanic provinces in Libya, in a trend NNW to SSE. 
The study area represents the north-eastern part of Jabal Al Haruj al Aswad and is limited by latitudes $27^{\circ} 00^{\prime} 00^{\prime \prime} \mathrm{N}$ to $27^{\circ} 50^{\prime} 00^{\prime \prime} \mathrm{N}$ and longitudes $18^{\circ} 00^{\prime} 00^{\prime \prime} \mathrm{E}$ to $18^{\circ} 39^{\prime} 58.5^{\prime \prime} \mathrm{E}$ (Figure 2). The landscape of the study area is characterized by a hilly morphology with frequent steep lava fronts, pressure ridges and blocky lava fields, with transverse intermittent desert sands dotted by balta (kipukas), but in other parts the relief is more or less flat.

Field geological mapping was carried out using Landsat TM multispectral satellite imagery with a scale of 1: 100,000 , which can be fitted with any kind of coordinate system with an accuracy of at least $20 \mathrm{~m}$, using a few calibration points that were measured during the fieldwork by GPS (Figure 2). This image formed the base of the geological map completed with altitude contours, locality names and other relevant data, Peregi et al. [5].

As the study area is remote, rather inaccessible and extremely difficult terrain, the fieldwork was carried out with the Industrial Research Centre (IRC), Tripoli as part of the geological mapping project of SE Libya in March 2006. During fieldwork, systematic field traverses were carried out by researchers equipped with GPS units, digital cameras and so on.

During the field trip, all basaltic exposures were investigated. About 109 samples were carefully collected from the flows for each phase (Figure 3). Of these, 42 samples were prepared for thin sections, and 29 samples were chosen for the major and trace element analysis using the XRF (Philips 2440) technique carried out by the Central Laboratories of the Egyptian Geological Survey, Cairo.

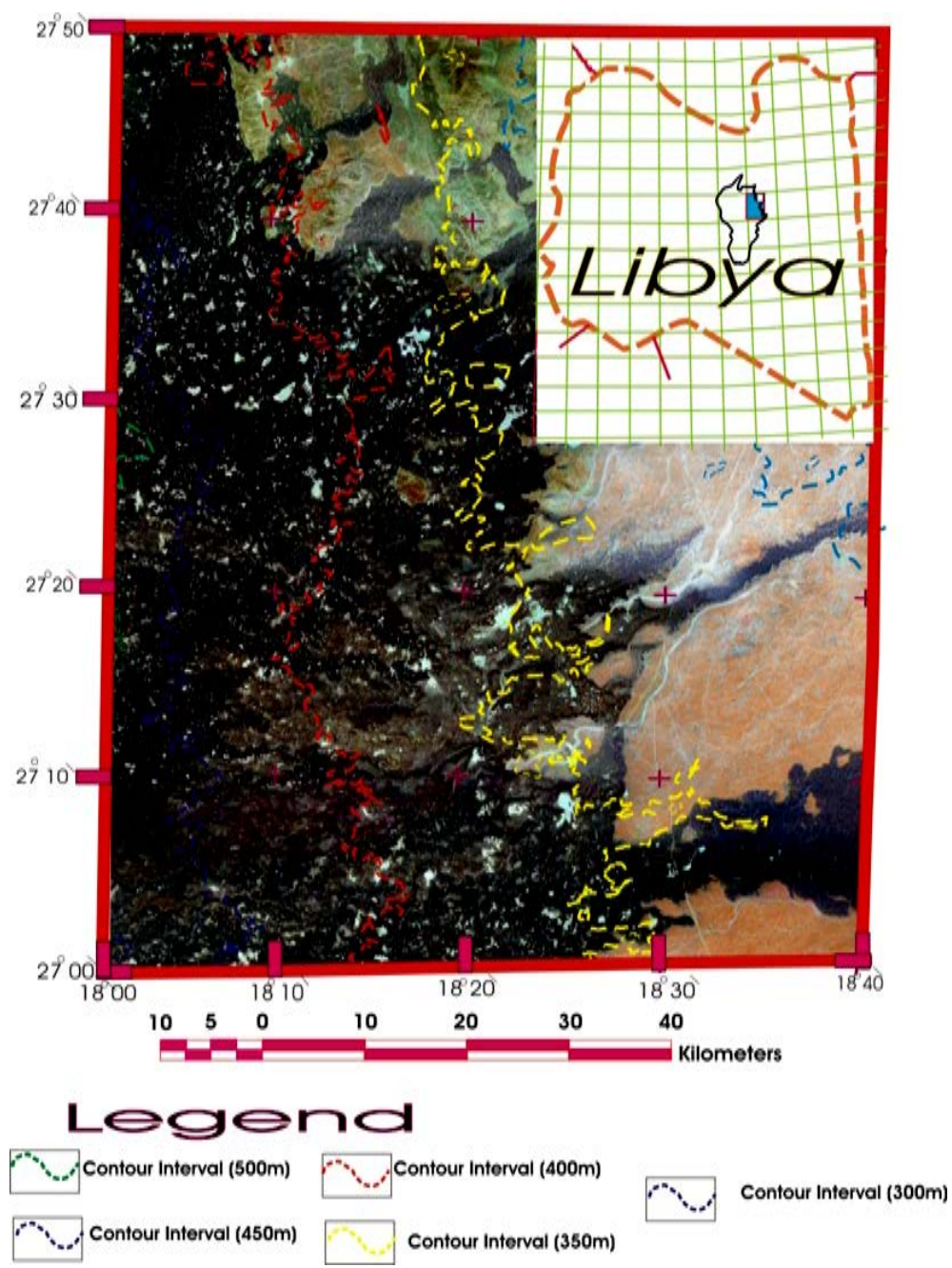

Figure 2. Landsat image of the north-eastern part of Al Haruj al Aswad, mosaiced and overlain by topographic contour lines to depict the spatial distribution of major volcanic phases. 


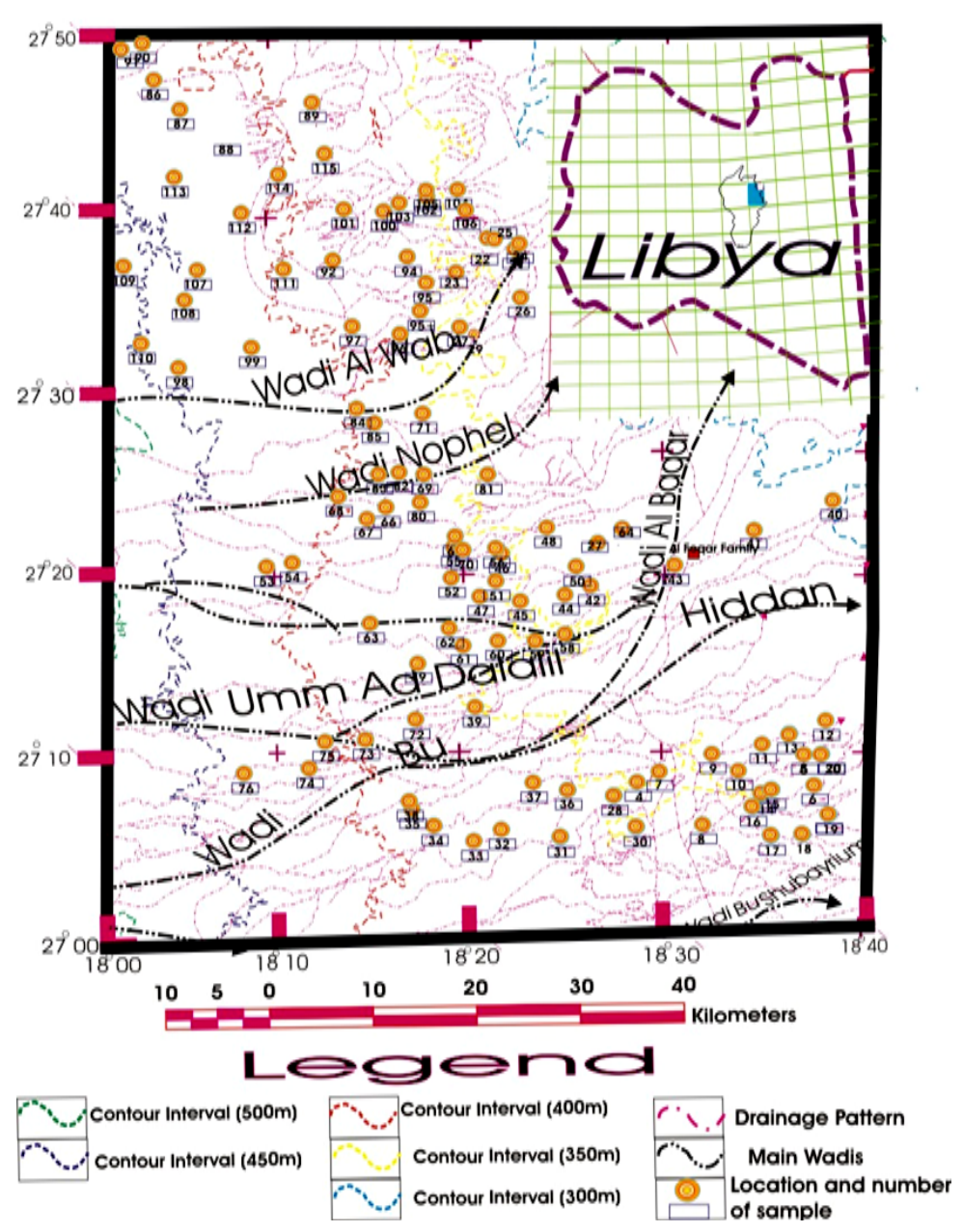

Figure 3. Topographic contour map showing the main wadis and their drainage patterns, as well as the localities of the samples.

The drainage patterns developed on the surfaces of the basaltic lava flows show systematic changes with the increasing age of basaltic lava flows, Busrewil and Suwesi [2]. The Landsat TM image shows the drainage pattern frequently increases in density as the basaltic rock become progressively older or decreases as the flow become younger, as in Phase 5, El-Shaafi [6]. The area investigated is cut by many wadis flowing from west and south-west towards the east and south-east, most of them starting from the central part of Al Haruj Al Aswad, outside the study area.

In the study area, the most common type of drainage pattern is dendritic, which is a well-integrated pattern formed by the main wadis (Wadi Bu al Hiddan, Wadi Al Baqar and Wadi Umm daladil), with tributaries branching and rebranching in several directions without systematic arrangement, Busrewil and Suwesi [2].

The core of southern Libya was formed by Precambrian basement massifs represented by Jabal Awayant, the Tibesti massif and the Tihemboka Arch, which are parts of the Nile craton, East Saharan craton and Pan-African remobilized belt, Hallett [7].

During the Pan-African orogeny, the rocks within these terrains were uplifted and accompanied by intense deformation, shearing and metamorphism. The main phase of the pan-African orogeny was completed about 540 Ma, followed by post-orogenic magmatism until about $440 \mathrm{Ma}$, interpreted by Klitzsch [8] as the early manifestation of the Caledonian orogeny.

This event was accompanied by the formation of NNW-SSE trending extensional structures more than a thousand km long such as the Kalanshio trough, south Haruj uplift, Dor el Qussah trough and the Tripoli-Tibesti uplift, among others. These directly affected the geological history of Al Haruj al Aswad and al Abiyad, Peregi et al. [5]. 
Toward, the Haruj uplift, the Palaeozoic geological development is much less clear than in the Dor el Qussah trough, as this area is covered by the volcanic pile of Al Haruj al Aswad. Following the Pan-African orogeny, Lower Paleozoic sediments were deposited and probably overlain by the Middle-Upper Devonian and Carboniferous sedimentary formations, Hallett [7].

After the Hercynian orogeny (Figure 4) almost the entire Kalanshio trough became part of Sirt Arch and most of the Palaeozoic sequence was destroyed by erosion, Peregi et al. [5].

The first data on the radiometric age lava of Jabal Al Haruj was given by Pesce [10], who considered it to be Late Pliocene based on K/Ar determination.

Ade Hall et al. [11] published the first results of a detailed K/Ar whole rock radiometric age determination survey combined with a palaeomagnetic measurement of the Al Haruj volcanic area. The measurements give an interval from 6 to $0.4 \mathrm{Ma}$ for the formation of the volcanic rocks, Less et al. [12], with the majority of the flows being younger than 2.2 Ma. The modern study of age dating by Peregi et al. [5] was based on K/Ar analyses of 20 basalt samples carried out at Atomki in Hungary.

It shows that the volcanic activities continued from $5.27 \mathrm{Ma}$ to about $0.1 \mathrm{Ma}$, which means that they can be assigned to the Pliocene and Pleistocene epochs, taking the limit of the Miocene at $5.3 \mathrm{Ma}$ according to the Global Standard Stratigraphic Age (Table 1).

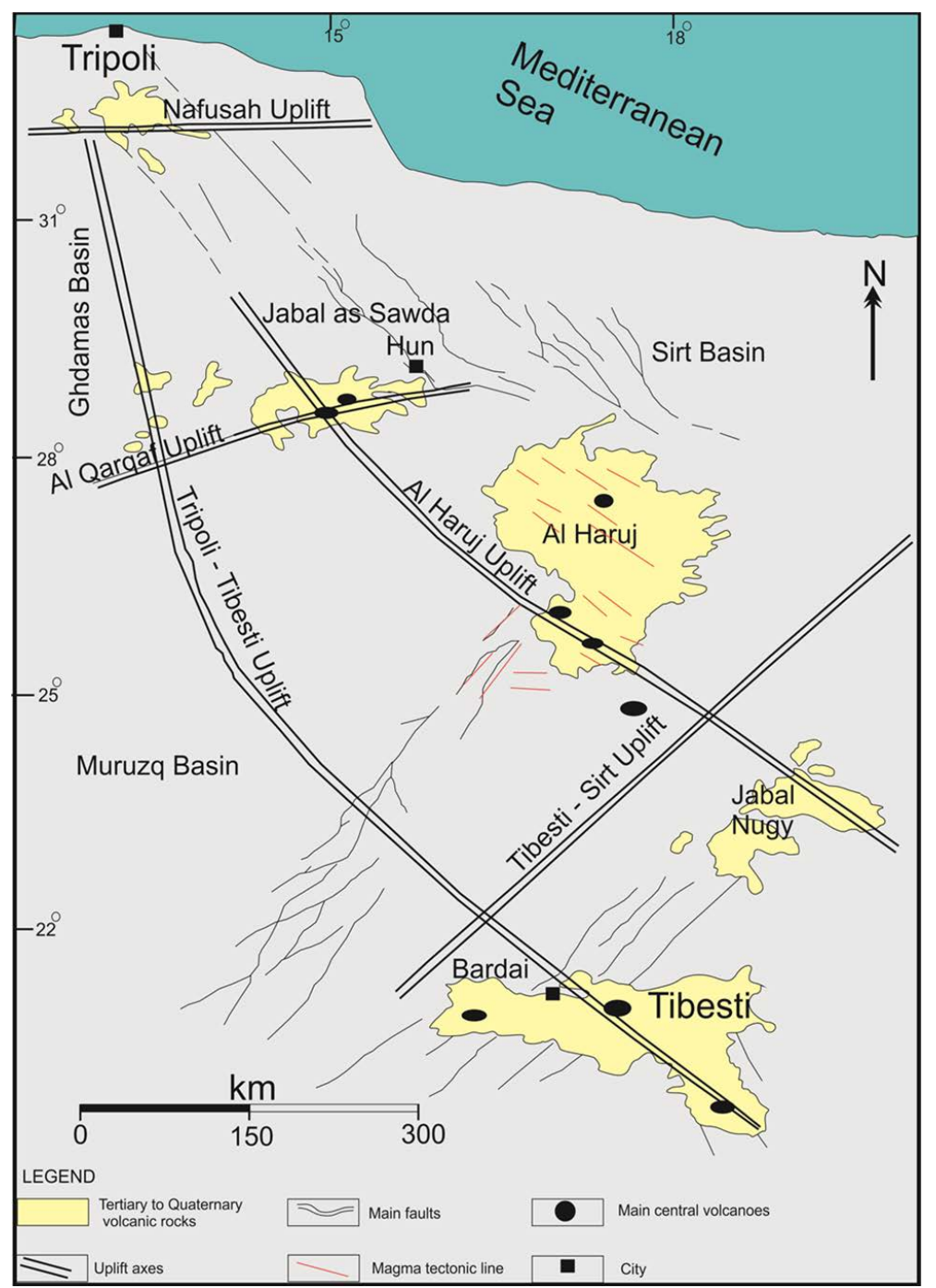

Figure 4. The volcanic distribution and relation between NW trending (Caledonian) and NE trending (Hercynian) structural elements and the volcanic eruptions, after Woller and Feduick [9]. 
Table 1. Age of the volcanic phases according to radiometric age determinations and paleomagnetic measurements, and their geological interpretation, Peregi et al. [5].

\begin{tabular}{ccc}
\hline Volcanic Phases & Age in Ma & Presumed geological age \\
\hline 6 & $0.10-0.30$ & Late to Middle Pleistocene \\
5 & $0.30-0.50$ & Middle Pleistocene \\
4 & $0.50-1.22$ & Early to Middle Pleistocene \\
3 & $1.22-1.77$ & Early Pleistocene \\
2 & $1.77-2.24$ & Late Pliocene \\
1 & $2.73-5.27$ & Early to late Pliocene \\
\hline
\end{tabular}

From this review it is clear that the study area has not been thoroughly studied so far, El-Shaafi [6], except for the work of Klitzsch [13], which deals with the regional geological mapping of Jabal Al Haruj (Al Abiyad and $\mathrm{Al}$ Aswad), showing the distribution of six major phases and one minor phase.

On the other hand, Farahat et al. [14] mentioned that the Libyan low volcanicity rift is probably related to the large Afro-Arabian rift system that extends from Turkey to Mozambique. This rift system is thought to have developed episodically rather than continuously. Age dating has suggested that the eastern rift in Kenya has evolved in three major pulses as follows: Eocene (44 - $38 \mathrm{Ma}$ ), Middle Miocene (16 - $11 \mathrm{Ma}$ ) and Pliocene to Pleistocene (5 - $0 \mathrm{Ma})$. These ages are generally contemporaneous with the ages of the Libyan volcanics; for example, Jabal Al Haruj was contemporaneous with the third pulse and the Gharyan volcanic province with the three pulses.

The linear trend of the Libyan volcanics and their decrease in age towards the SSE is probably caused by intraplate extensional stresses. Continental rifting is normally associated with asthenospheric upwelling and high heat flow, but there is some controversy as to the mechanism involved, Farahat et al. [14]. It is difficult to decide whether the volcanic activity in Jabal Al Haruj was related directly to an uprising mantle plume (active rifting) or to the extension of the lithosphere (passive rifting), Peregi et al. [5]. The present work deals mainly with the different exposures in the study area. The exposed strata range in age from the Lower Miocene to Upper Pleistocene. Stratigraphically, the basalt flows in the study area disconformably overlie the older sequence of sedimentary rocks. The investigated volcanic rocks are overlay Maradah Formation and have a varied morphology, implying that they are produced episodically in consistency with available age data of 5.3 to 0.10 Ma from the Upper Miocene to Upper Pleistocene, Peregi et al. [5].

The study area is not mountain range but rather a vast plateau. The commonest type of volcanic occurrence is represented by basalt lava flows, which shaped the present morphology. Generally, the central eruption of these lava flows are most markedly shown in the central part of Al Haruj, while elsewhere it is on its margins. In contrast, and despite the study area being located at the margin of Al Haruj, it is difficult to locate these features.

The Jabal Al Haruj Al Aswad volcanic province is characterized by the prevalence of lava of an effusive form rather than an intrusive form. Although some of the lava may have come from central volcanoes, the great volume of lava and the remarkable uniformity in thickness of individual lava sheets over a wide area indicate that the lava must have extruded from numerous fissures, Farahat et al. [14]. In the area investigated, plateau basalts are the most widespread; they are composed of several lava flows, whose thickness does not exceed several metres. Five major volcanic phases of different field appearances (including weathering surface, morphology, and primary structures) have been documented in the investigated area in the north-eastern part of $\mathrm{Al} \mathrm{Haruj} \mathrm{Al}$ Aswad province (Figure 5).

\section{Petrography}

The petrographic characteristics of the Tertiary volcanic rocks of Jabal Al Haruj (al Aswad and al Abiyad) have been studied by many authors, among them Busrewil and Suwesi [2], Less et al. [12], Peregi et al. [5], Vesely [15] and Woller [16].

In the present study, 109 samples from the investigated area were described, and on the basis of preliminary 


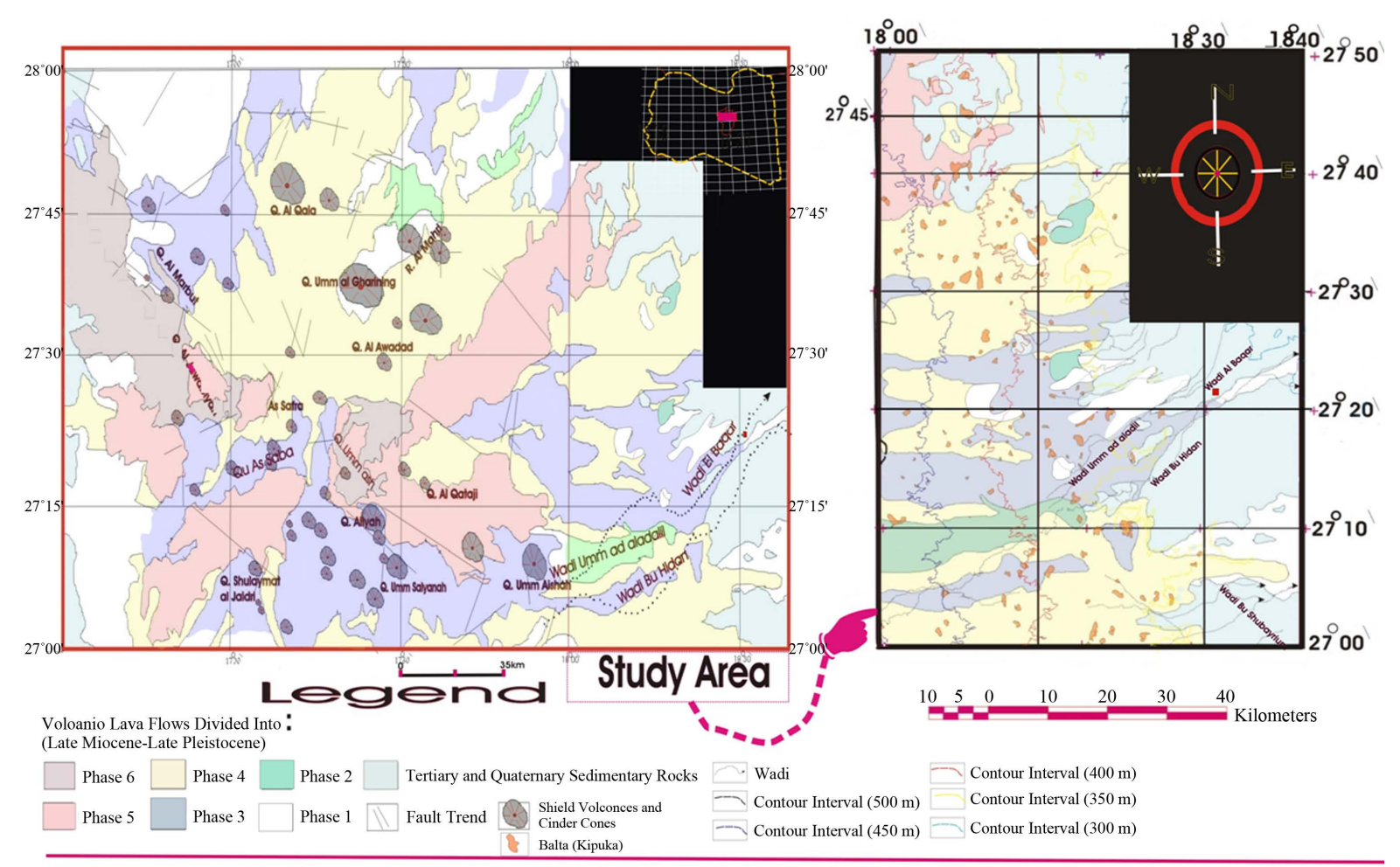

(a)

(b)

Figure 5. Geological map showing (a) The central part of al Haruj al Aswad, studied by Busrewill and Suwesi [2] and the north-eastern part of al Haruj al Aswad (study area) investigated in the present work; (b) Magnified geological map of the study area.

petrographic descriptions 42 samples were selected for detailed petrographic study. Modal analyses of the various phases of basaltic rocks were carried out using an electronic point counter (Model E). The results of the modal analyses are presented in Table 2.

Microscopically, the rock samples are variably vesicular, amygdaloidal and almost medium grained and subordinately fine grained. Some of the samples are characterized by the presence of variable amounts of glass in the groundmass, but most of them are holocrystalline with an intergranular to intersertal texture.

The phenocrysts consist predominately of olivine and a subordinate amount of clinopyroxene, while plagioclase is not found. Olivine phenocrysts form about $7 \%$ to $21 \%$ by volume of the rock and occur as subhedral and sometimes as euhedral crystals without zoning; therefore, they indicate the presence of enough time to equilibrate with the melting at mantle depth.

Olivine phenocrysts sometimes occur as clots displaying a glomerporphyritic texture. Pyroxene phenocrysts were found in some samples as elongated and prismatic crystals. The matrix consists mostly of medium grained plagioclase laths, olivine, clinopyroxene (augite) and opaque minerals (probably ilmenite).

Plagioclase laths are the most abundant, representing $32 \%$ to $48 \%$ of the matrix in most of the studied samples. They are both pilotaxitically and randomly arranged, and sometimes they exhibit a trachytic arrangement around olivine phenocrysts. Cryptocrystals fill the interstices between plagioclase laths. Some samples showed vesicles filled with carbonate secondary minerals.

The different textures and mineralogical composition of basaltic rocks of each phase are described in more detail in El-Shaafi [6]. A detailed petrographic description of Phase 1 follows.

\section{Phase 1}

This phase represents the earliest volcanic activity in the area. The lava flow of this phase is highly weathered, but although weathered in the field, the plagioclase and pyroxene (augite) are nearly fresh under the microscope.

Olivine phenocrysts are partially altered to iddingsite, which has stained the rocks a red colour. The most 
Table 2. Model analyses of representative basaltic rocks from north-eastern part of Al Haruj al Aswad.

\begin{tabular}{ccccccccc}
\hline $\begin{array}{c}\text { Phase } \\
\text { Number }\end{array}$ & $\begin{array}{c}\text { Sample } \\
\text { Number }\end{array}$ & $\begin{array}{c}\text { Olivine } \\
\%\end{array}$ & $\begin{array}{c}\text { Clino-Pyroxene } \\
\%\end{array}$ & $\begin{array}{c}\text { Plagioclase } \\
\%\end{array}$ & Opaque $\%$ & Glass \% & Carbonate \% & Texture Type \\
\hline 1 & 17 & 18.57 & 21.76 & 37.28 & 12.96 & 5.69 & 3.74 & Porphyritic Intergranular \\
1 & 54 & 21.55 & 24.84 & 39.14 & 9.09 & 2.21 & 3.17 & Porphyritic Intergranular \\
2 & 23 & 13.77 & 19.55 & 41.40 & 8.11 & 8.20 & 8.97 & Porphyritic Intergranular \\
2 & 95 & 12.29 & 15.01 & 37.41 & 7.28 & 18.30 & 9.71 & Intersertal, Amygdaloidal \\
3 & 35 & 15.87 & 24.11 & 45.31 & 8.21 & 3.99 & 2.51 & Porphyritic Intergranular \\
3 & 51 & 16.21 & 20.01 & 43.72 & 6.25 & 10.41 & 3.41 & Intergranular to Intersertal \\
3 & 68 & 16.45 & 19.92 & 48.46 & 4.29 & 7.48 & 3.40 & Intergranular to Intersertal \\
4 & 30 & 18.01 & 19.56 & 46.09 & 5.02 & 10.71 & 0.61 & Intergranular to Intersertal \\
4 & 69 & 19.10 & 21.14 & 47.16 & 4.77 & 3.68 & 4.15 & Intergranular to Intersertal \\
4 & 108 & 6.71 & 26.88 & 46.43 & 2.56 & 13.86 & 3.56 & Intergranular to Intersertal \\
5 & 86 & 7.83 & 12.99 & 32.92 & 0.78 & 42.76 & 2.72 & Intersertal to Hyalo Ophitic \\
\hline
\end{tabular}

common texture of the rocks of this phase is an intersertal to intergranular texture (Plate 1). The intergranular texture is one in which the dimensions of the plagioclase and pyroxene are similar and the glass is relatively minor, which grades into intersertal when interstitial glass is a significant component.

The rocks are composed mainly of plagioclase, clinopyroxene and olivine. Opaque minerals that might be ilmenite are present as accessory minerals. Reddish brown iddingsite and carbonate minerals are secondary products. Volcanic glass is also observed. The detailed petrography of Phase 1 as follows:

A-Plagioclase is the most abundant, occurring in the form of laths of lamellar twinned crystals, ranging in size from 0.40 to $0.95 \mathrm{~mm}$ in length and from 0.10 to $0.20 \mathrm{~mm}$ in width, and occurring in the form of irregular grains. The interference colour of plagioclase laths of the first order is grey to straw-yellow with broad twin lamellae. Generally, the relief of plagioclase is quite high, so this is a calcium-rich plagioclase, Mackenzie and Adams [17].

Plagioclase crystals contain opaque crystals as inclusions, El-Shaafi [6]. There is more than one generation of plagioclase: the first generation produced small plagioclase crystals which occur as inclusions within clinopyroxene; the second produced lath-shaped crystals with lamellar twinning. Optical measurements indicate that the plagioclase compositions are generally in the range of An $61 \%$ to An $72 \%$ (labradorite-bytownite), which is characterized by broad twin lamellae under cross-polarized light. Laths of plagioclase are commonly seen to flow around phenocrysts and form a trachytic texture, which may have resulted from the flow of the magma before solidification, El-Shaafi [6].

B-Olivine is the third of the abundant minerals which occur in the euhedral to subhedral form, or as rounded crystals. The olivine crystals range in size from 0.40 to $1.2 \mathrm{~mm}$ in length and from 0.40 to $0.70 \mathrm{~mm}$ in width, although rounded crystals averaging $0.38 \mathrm{~mm}$ diameter are recorded. It is recognized in the examined samples by its colourless, high relief and high birefringence, and it is commonly traversed by randomly oriented cracks.

Olivine phenocrysts are partially or completely converted into a strongly coloured reddish brown along their cracks and boundaries, known as iddingsite, El-Shaafi [6]. Multiple grains as clusters of adhering phenocrysts of olivine were observed and formed a glomerporphyritic texture, El-Shaafi [6]. Skeletal crystals of olivine were observed, where the edges and corners grow more rapidly than the faces, El-Shaafi [6]. More than one generation of olivine were observed: the first generation were the phenocrysts, which appear as highly cracked crystals, whereas the second generation produced small scattered crystals associated with clinopyroxene (augite) occupying the spaces between the plagioclase.

C-Clinopyroxene occurs in a subhedral to anhedral form, and as prismatic crystals varying from $0.35 \mathrm{~mm}$ to $0.85 \mathrm{~mm}$ in length and from 0.10 to $0.60 \mathrm{~mm}$ in width, which have a maximum extinction angle of $42^{\circ}$ to $51^{\circ}$. They are identified as clinopyroxene (augite). Augite crystals are colourless to pale brown, and brown in some 

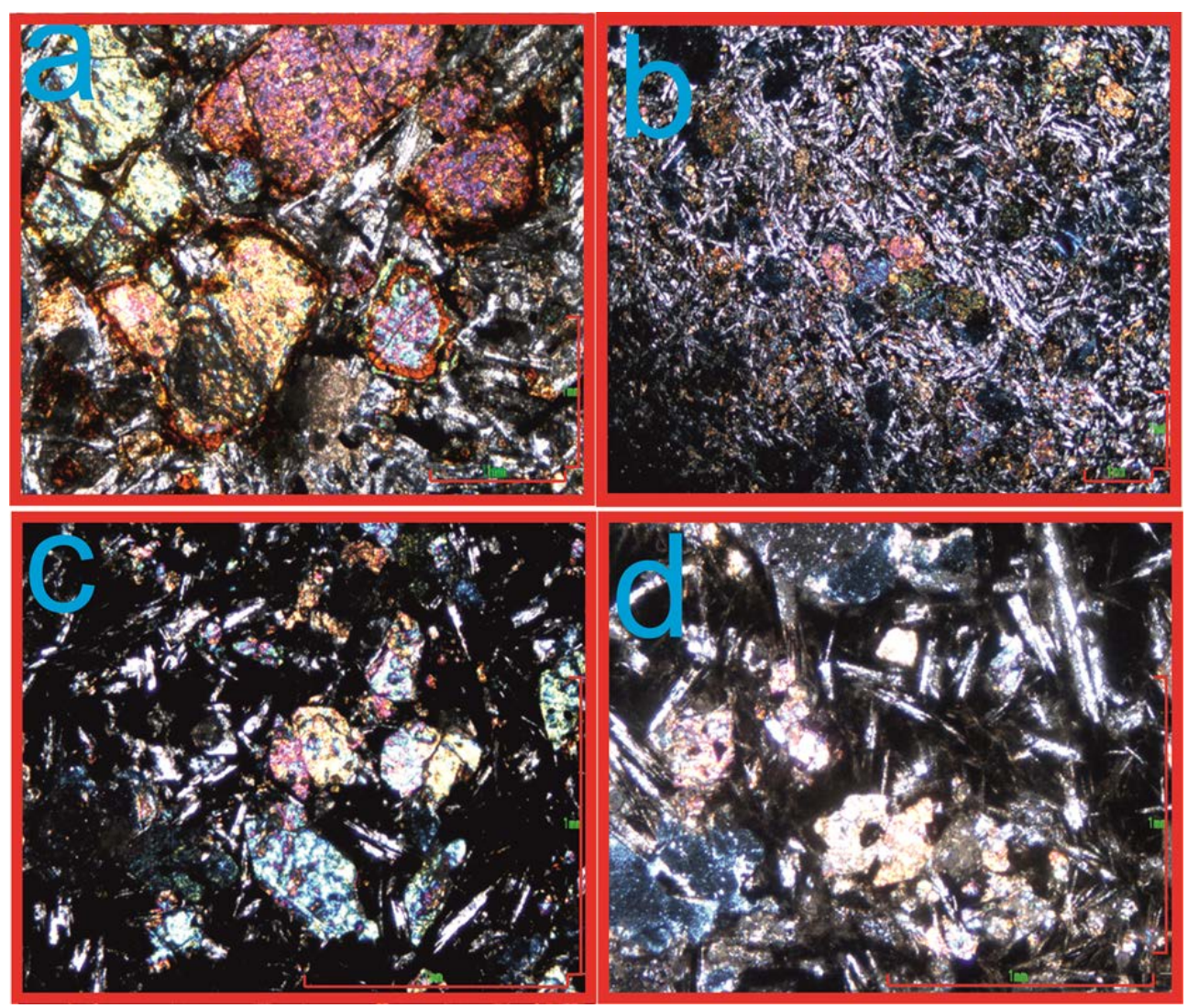

Plate 1. Microphotographs of olivine basalt of phase one, showing fine-grained rock with microphenocrysts of olivine (highest interference colour) and clinopyroxene (augite). The groundmass is composed of olivine, laths shaped of plagioclase and clinopyroxene (augite), with roughly rectangular crystals of opaque minerals. A higher magnification view reveals the olivine crystals and laths of plagioclase in more detail. Sometimes clinopyroxene crystals occur as a large poikilitic plate, enclosing several plagioclase and olivine crystals and forming an ophitic texture.

specimens. Rarely, the crystals show simple twining. The crystals are generally fresh, but may be slightly altered along the cracks and cleavages. Some of them contain inclusions of plagioclase and opaque minerals (most probably ilmenite).

A subophitic texture is well developed in the rock, where plagioclase laths are partly enclosed with clinopyroxene, whereas the carbonate (most probably calcite) iddingsite occurs and secondary constituents.

D-Opaque minerals are present in considerable amounts, being in the form of discrete crystals that exhibit various forms. They are generally of a smaller size than associated silicate minerals, where they range in length from $0.05 \mathrm{~mm}$ to $0.20 \mathrm{~mm}$ and from $0.035 \mathrm{~mm} 0.10 \mathrm{~mm}$ in breadth, El-Shaafi [6]. They are the primary constituents, being crystallized straight from the melt.

E-Volcanic glass is present in some of the examined samples, but in small amounts between the plagioclase laths, manifesting an intersertal texture.

F-Carbonate occurs plentifully in some samples in the interstitial spaces and it fills irregular vesicles and forms an amygdaloidal texture, El-Shaafi [6]. Carbonate minerals have higher birefringence, so that when viewed in cross-polarized light (XPL), they do not show interference colours; instead, they show delicate pastel shades of colours because of the extreme birefringence, which reaches up to the sixth order of interference colour.

G-Iddingsite and bowlingite. In certain samples of the olivine basalts of Phase 1, the olivine phenocrysts are partially or completely altered into a strongly coloured yellowish or reddish brown mineral called iddingsite, El-Shaafi [6]. Bowlingite may be similar to iddingsite, but bowlingite is more strongly coloured than iddingsite and is rich in red colour, Hatch et al. [18]. 


\section{Geochemistry}

The geochemistry of Late Miocene-Pleistocene basaltic rocks of Al Haruj area has previously been treated by investigators. Busrewil and Wadsworth [1], made the first attempt to undertake a preliminary study of chemical data on $\mathrm{Al} \mathrm{Haruj} \mathrm{volcanics.} \mathrm{These} \mathrm{authors} \mathrm{indicated} \mathrm{that} \mathrm{the} \mathrm{rocks} \mathrm{of} \mathrm{Al} \mathrm{Haruj} \mathrm{belong} \mathrm{to} \mathrm{the} \mathrm{alkali} \mathrm{olivine} \mathrm{basalt}$ association, ranging in composition from basalts to hawaiites. Vesely [15] revealed that the rocks there are composed of alkaline to tholeiitic basalts. Busrewil and Suwesi [2] suggested the clustering of Al Haruj volcanics into four trends of differentiation, which may be described as follows: alkali basalts of the block lava series, alkali basalts of the main lava series, subalkali basalts and dolerites.

Peregi, et al. [5] studied the geochemistry of basaltic rocks from Al Haruj al Abiyad; they concluded that they were formed in an intracontinental (within plate) tectonic setting. They classified them into three main mafic groups based on their chemical composition: basanites, alkali basalts and olivine tholeiites. Moreover, they originated from different degrees of partial melting of a similar mantle source. The basanite magmas could have been formed by $0.5 \%$ to $1 \%$ partial melting, whereas the primary magmas of the alkaline basalts could have been generated by $2 \%$ to $6 \%$ partial melting, Peregi, et al. [5].

The present study is an attempt to identify the geochemical characteristics of the Late Miocene-Pleistocene basaltic rocks of the NE part of Al Haruj al Aswad, evaluate the chemistry of these rocks within the framework of established magma types and determine the tectonic environment of their eruption. A major step in the development of evaluating the studied basaltic rocks involves choosing a method that enables rapid and accurate analysis to be conducted of major and trace elements present at low concentrations in silicate materials.

Twenty-nine samples were chemically analysed using X-ray fluorescence (Philips 2404) at the Central Laboratories of the Geological Survey of Egypt to determine the major and trace elements of the chemically analysed samples (Figure 6). Details of the analytical procedure and precision can be found in Appendix 1 of El-Shaafi [6]. The geochemical data of the 29 samples and the CIPW norms are presented in Table 3.

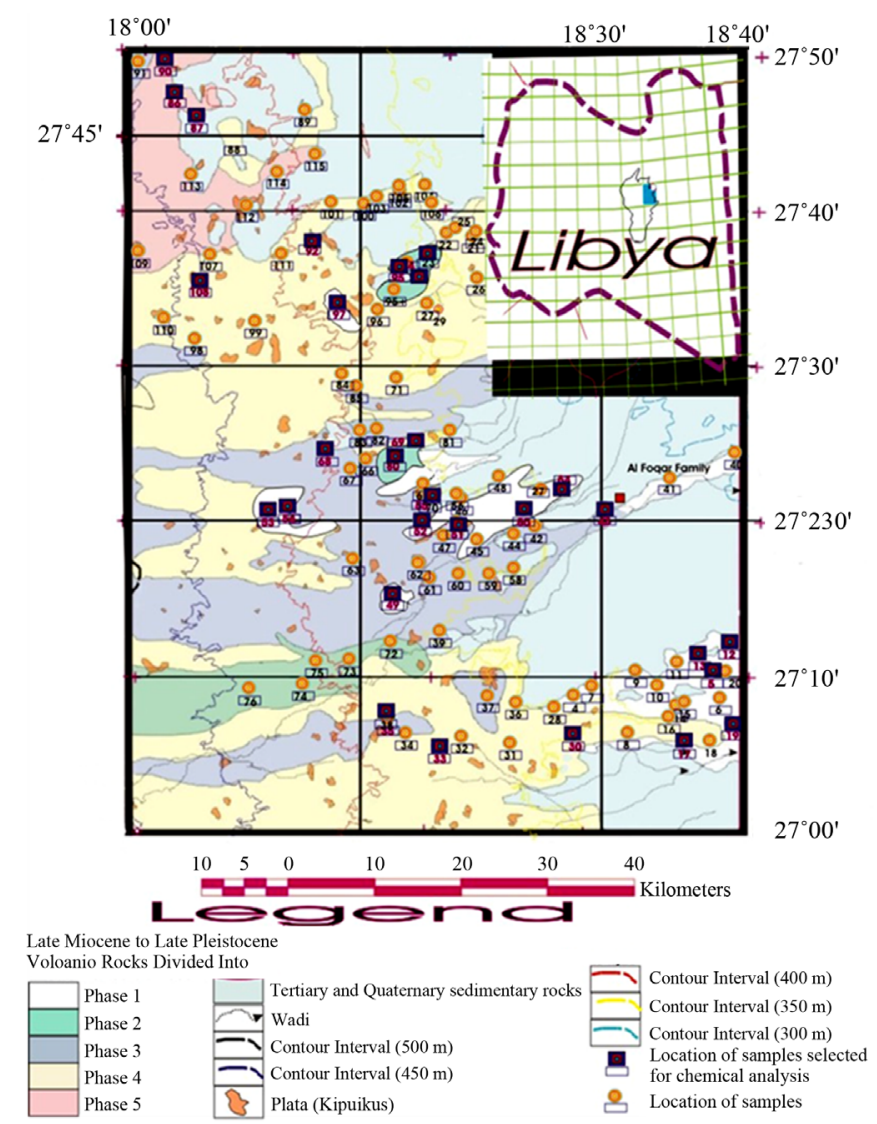

Figure 6. Geological map of the investigated area showing the sample localities of all collected samples during field inspection and location of samples selected for chemical analysis. 
Table 3. Representative analyses of the major elements (wt\%), trace elements (in ppm) and (CIPW) norms of the studied basaltic rocks. CIPW normative components are calculated for the volatile free data normalized to $100 \%$. $\mathrm{Fe}_{2} \mathrm{O}_{3}$ and $\mathrm{FeO}$ were estimated based on ratio of $\mathrm{Fe}_{2} \mathrm{O}_{3} / \mathrm{FeO}=0.2$.

(a)

\begin{tabular}{|c|c|c|c|c|c|c|c|c|c|c|c|c|c|c|c|c|c|}
\hline \multicolumn{18}{|c|}{ Representative analyses of the major elements (wt\%) of Jabal al Haruj al Aswad } \\
\hline & ntrusion & $\mathrm{SiO}_{2}$ & $\mathrm{TiO}_{2}$ & $\mathrm{Al}_{2} \mathrm{O}_{3}$ & $\mathrm{Fe}_{2} \mathrm{O}_{3}$ & $\mathrm{FeO}$ & $\mathrm{MnO}$ & $\mathrm{MgO}$ & $\mathrm{CaO}$ & $\mathrm{Na}_{2} \mathrm{O}$ & $\mathrm{K}_{2} \mathrm{O}$ & $\mathrm{P}_{2} \mathrm{O}_{5}$ & LOI & $\mathrm{SO}_{3}$ & $\mathrm{Cl}$ & Sum & Total I \\
\hline DN19 & lase 1.1 & 49.85 & 1.79 & 15.81 & 1.65332 & 8.2666 & 0.15 & 6.49 & .26 & 3.65 & 0.98 & 0.25 & 0.01 & 0.14 & 0.13 & 98.43 & 0.84 \\
\hline DN49 & hase 1.1 & & 1.55 & & 1.68688 & 8.4344 & 0.16 & 6.38 & 9.92 & 3.38 & 0.74 & 0.22 & 1.17 & 0.85 & 0.16 & 98.421 & 11.06 \\
\hline DN50 & hase 1.1 & 48.13 & 1.62 & 15.77 & 1.62282 & 8.1141 & 0.16 & 5.54 & 10.02 & 3.6 & 0.73 & 0.19 & 1.39 & 1.57 & 0.24 & 98.697 & 10.64 \\
\hline DN52 & Phase 1.1 & & 1.87 & 15.63 & 1.53 & & & & 12.33 & 3.45 & 0.96 & 0.32 & & 0.31 & 0.11 & 99.32 & 10.13 \\
\hline DN53 & Phase 1.1 & 47.63 & 1.73 & 15.11 & 1.67467 & 8.3734 & 0.16 & 6.56 & 10.17 & 3.39 & 0.97 & 0.31 & & 1.69 & 0.18 & 98.718 & 0.98 \\
\hline DN54 & hase 1.1 & 50.06 & 1.64 & 16.33 & 1.57554 & 7.8777 & 0.16 & 6.02 & 9.37 & 3.7 & 0.91 & 0.23 & & 0.1 & 0.2 & 98.723 & 10.33 \\
\hline DN97 & hase 1.1 & 50.18 & 1.64 & 16.41 & & 8.3581 & 0.16 & 5.72 & 9.36 & 3.96 & 0.68 & 0.19 & 0.01 & 0.18 & 0.16 & 98.68 & 10.96 \\
\hline DN64 & Phase 1.1 & 46.93 & 1.56 & 15.27 & 1.67162 & 8.3581 & 0.16 & 5.7 & 11.82 & 3.24 & 0.69 & 0.28 & & 1.81 & 0.1 & 98.622 & 10.96 \\
\hline DN5 & Phase 1.1 & 8.45 & 1.64 & 15.08 & 1.68993 & 8.4496 & 0.16 & 6.7 & 10.4 & 3.22 & 0.77 & 0.23 & 0.94 & 0.69 & 0.26 & 98.68 & 11.08 \\
\hline DN12 & Phase 1.1 & 50.85 & 1.83 & 15.67 & 1.62892 & 8.1446 & 0.15 & 5.59 & 9.21 & 3.65 & 0.79 & 0.14 & & 0.41 & 0.23 & 98.694 & 10.68 \\
\hline DN13 & lase 1.1 & 50.76 & 1. & 16.35 & & & 0.15 & 5.56 & 9.46 & 3.82 & 0.95 & & & 0.12 & 0.16 & 73 & 0.37 \\
\hline DN17 & Phase 1.1 & 47.66 & 1.61 & 14.44 & 1.62282 & 8.1141 & 0.15 & 6.65 & 10.9 & 3 & 0.54 & 0.15 & & 1.9 & 0.2 & 98.527 & 10.64 \\
\hline DN23 & Phase n.2 & 8.25 & 1.88 & 15.81 & 1.61062 & 8.0531 & 0.16 & 5.26 & 10.46 & 3.55 & 0.81 & 0.31 & 1.78 & 0.7 & 0.17 & 98.804 & 10.56 \\
\hline DN80 & Phase n.2 & 47.89 & 1.68 & 14.63 & & & 0.15 & 6.95 & 10.44 & 3.29 & 0.78 & 0.22 & 1.83 & 0.91 & .15 & 98.712 & 10.7 \\
\hline DN94 & Phase n.2 & 49.95 & 1.71 & 17.5 & & 7.6031 & 0.15 & 4.75 & 10.56 & 4 & 0.54 & 0.27 & 0.19 & 0.09 & .05 & 98.884 & 97 \\
\hline DN95 & Phase n.2 & 50.06 & 1.87 & 15.74 & 1.60451 & 8.0226 & 0.16 & 6.05 & 9.54 & 3.6 & 0.86 & 0.23 & 0.39 & 0.53 & 0.12 & 98.777 & 10.52 \\
\hline DN35 & Phase n.3 & 48.75 & 2.07 & 15.22 & & 8.5 & 0.16 & 6.32 & & & 0.96 & 0.37 & 0.19 & 0. & 0.1 & 98.753 & 11.27 \\
\hline DN43 & Phase n.3 & 4 & 1.57 & 15.81 & & .748 & 0.15 & 5.66 & 10.38 & 3.53 & 0.77 & 0.19 & 1.26 & 1.67 & 0.18 & 98.768 & 0.1 \\
\hline DN51 & Phase n.3 & 48.48 & & 14.88 & & & 0.15 & 7.51 & & 3 & & 0.24 & 0.64 & 1.26 & 0.21 & & 10.62 \\
\hline DN55 & Phase n.3 & 50.12 & 1.83 & 16.62 & 1.61062 & 8.0531 & 0.16 & 4.71 & 10.34 & 3.85 & 0.74 & 0.29 & 0.15 & 0.14 & 0.09 & 98.704 & 10.56 \\
\hline DN68 & Phase n.3 & 49.51 & 1.87 & 15.16 & 1.63502 & 8.1751 & 0.17 & 7.66 & 9.23 & 3.45 & 0.89 & 0.34 & 0.12 & 0.39 & 0.07 & 98.67 & 10.72 \\
\hline DN30 & Phase n.4 & 51.63 & 1.82 & 16.54 & 1.45047 & 7.2523 & 0.15 & 5.36 & 8.97 & 4.03 & 1.06 & 0.3 & 0.01 & 0.05 & 0.13 & 98.753 & 9.51 \\
\hline DN33 & Phase n.4 & 49.45 & 1.62 & 14.80 & 1.63654 & 8.1827 & 0.15 & 7.00 & 10.23 & 2.96 & 0.56 & 0.14 & 1.01 & 0.79 & 0.18 & 98.709 & 10.13 \\
\hline DN69 & Phase n.4 & 49.65 & 1.68 & 16.25 & 1.59689 & 7.9844 & 0.16 & 5.82 & 10.24 & 3.75 & 0.83 & 0.26 & 0.13 & 0.16 & 0.18 & 98.691 & 10.47 \\
\hline DN92 & Phase n.4 & 51.60 & 1.81 & 15.65 & 1.61977 & 8.0988 & 0.15 & 6.17 & 8.59 & 3.34 & 0.76 & 0.14 & 0.00 & 0.18 & 0.18 & 98.289 & 10.62 \\
\hline DN108 & Phase n.4 & 48.44 & 1.87 & 14.75 & 1.7189 & 8.5945 & 0.17 & 7.15 & 10.42 & 3.29 & 0.80 & 0.27 & 0.84 & 0.12 & 0.16 & 98.593 & 11.27 \\
\hline DN86 & Phase n.5 & 48.87 & 1.76 & 14.91 & 1.81499 & 9.075 & 0.17 & 7.35 & 10.09 & 2.99 & 0.68 & 0.15 & 0.11 & 0.49 & 0.20 & 98.66 & 11.90 \\
\hline DN87 & Phase n.5 & 48.77 & 2.06 & 14.07 & 1.91261 & 9.563 & 0.17 & 6.96 & 10.01 & 3.22 & 0.86 & 0.21 & 0.14 & 0.46 & 0.13 & 97.946 & 12.54 \\
\hline DN90 & Phase n.5 & 48.66 & 1.67 & 15.32 & 1.80737 & 9.0368 & 0.18 & 7.14 & 10.05 & 3.11 & 0.61 & 0.19 & 0.18 & 0.59 & 0.16 & 98.704 & 11.85 \\
\hline
\end{tabular}


(b)

\begin{tabular}{|c|c|c|c|c|c|c|c|c|c|c|c|c|}
\hline & \multicolumn{11}{|c|}{$\begin{array}{l}\text { CIPW norms of the studied Jabal al Haruj al Aswad basaltic rocks. CIPW normative components are calculated } \\
\text { for the volatile free data normalized to } 100 \% \mathrm{Fe}_{2} \mathrm{O}_{3} \text { and }\end{array}$} & \multirow[b]{2}{*}{ Ap } \\
\hline & Intrusion & $\mathrm{Q}$ & Or & $\mathrm{Ab}$ & An & $\mathrm{Ne}$ & Di & Hy & Ol & Mt & Il & \\
\hline DN19 & Phase 1.1 & & 5.91 & 30.89 & 23.86 & 0 & 17.1 & 2.03 & 12.72 & 2.44 & 3.46 & 0.58 \\
\hline DN49 & Phase 1.1 & & 4.55 & 28.6 & 25.045 & 0 & 19.3 & 0.45 & 13.8 & 2.54 & 3.06 & 0.53 \\
\hline DN50 & Phase 1.1 & & 4.49 & 29.04 & 24.714 & 0 & 20.4 & 0 & 11.8 & 2.46 & 3.23 & 0.46 \\
\hline DN52 & Phase 1.1 & & 5.79 & 20.94 & 24.326 & 4.87 & 29.3 & 0 & 7.6 & 2.28 & 3.63 & 0.74 \\
\hline DN53 & Phase 1.1 & & 5.79 & 24.45 & 23.147 & 1.85 & 21.6 & 0 & 13.41 & 2.52 & 3.42 & 0.74 \\
\hline DN54 & Phase 1.1 & & 5.5 & 31.31 & 25.262 & 0 & 16.4 & 3.2 & 10.98 & 2.32 & 3.19 & 0.56 \\
\hline DN97 & Phase 1.1 & & 4.08 & 33.51 & 24.992 & 0 & 16.9 & 1.155 & 12.28 & 2.46 & 3.17 & 0.44 \\
\hline DN64 & Phase 1.1 & & 4.25 & 21.79 & 25.084 & 3.22 & 27.3 & 0 & 10.01 & 2.51 & 3.1 & 0.67 \\
\hline DN5 & Phase 1.1 & & 4.73 & 27.25 & 24.419 & 0 & 21.7 & 0.84 & 13.04 & 2.54 & 3.21 & 0.56 \\
\hline DN12 & Phase 1.1 & & 4.79 & 30.89 & 24.04 & 0 & 17.4 & 10.84 & 4.4 & 2.41 & 3.55 & 0.32 \\
\hline DN13 & Phase 1.1 & & 5.73 & 32.32 & 24.659 & 0 & 17.5 & 3.3 & 9.53 & 2.33 & 3.29 & 0.46 \\
\hline DN17 & Phase 1.1 & & 3.37 & 25.39 & 24.34 & 0 & 24.7 & 3.29 & 10.11 & 2.48 & 3.23 & 0.37 \\
\hline DN23 & Phase n.2 & & 4.96 & 28.56 & 24.811 & 0.81 & 21.4 & 0 & 10.39 & 2.41 & 3.72 & 0.74 \\
\hline DN80 & Phase n.2 & & 4.85 & 26.25 & 22.847 & 0.88 & 23.5 & 0 & 13.17 & 2.45 & 3.34 & 0.53 \\
\hline DN94 & Phase $n .2$ & & 3.19 & 32.36 & 28.2 & 1.03 & 18.6 & 0 & 10.23 & 2.36 & 3.27 & 0.63 \\
\hline DN95 & Phase n.2 & & 5.2 & 30.46 & 24.248 & 0 & 18 & 0 & 8.68 & 2.36 & 3.63 & 0.56 \\
\hline DN35 & Phase n.3 & & 5.79 & 27.68 & 20.739 & 3.42 & 21.7 & 0 & 12.37 & 2.52 & 4.01 & 0.88 \\
\hline DN43 & Phase n.3 & & 4.79 & 28.12 & 25.019 & 1 & 21.6 & 0 & 11.17 & 2.33 & 3.11 & 0.46 \\
\hline DN51 & Phase n.3 & & 5.73 & 25.39 & 24.359 & 0 & 19.5 & 0 & 11.54 & 2.39 & 3.35 & 0.58 \\
\hline DN55 & Phase n.3 & & 4.43 & 32.58 & 25.882 & 0 & 19.8 & 0 & 9.31 & 2.35 & 3.53 & 0.67 \\
\hline DN68 & Phase n.3 & & 6.26 & 29.19 & 23.25 & 0 & 17 & 4.29 & 13.05 & 2.42 & 3.63 & 0.72 \\
\hline DN30 & Phase n.4 & & 6.38 & 34.1 & 23.91 & 0 & 15.5 & 6.03 & 6.91 & 2.13 & 3.51 & 0.7 \\
\hline DN33 & Phase n.4 & & 3.43 & 25.05 & 25.442 & 0 & 20.6 & 0 & 4.89 & 2.45 & 3.17 & 0.32 \\
\hline DN69 & Phase n.4 & & 5.02 & 30.06 & 25.055 & 0 & 20.1 & 0 & 11.68 & 2.35 & 3.25 & 0.6 \\
\hline DN92 & Phase n.4 & 0.65 & 4.61 & 28.26 & 25.465 & 0 & 13.6 & 20.07 & 0 & 2.39 & 3.51 & 0.32 \\
\hline DN108 & Phase n.4 & & 4.85 & 26.97 & 23.116 & 0.5 & 22.5 & 0 & 14 & 2.54 & 3.65 & 0.65 \\
\hline DN86 & Phase n.5 & & 4.08 & 25.3 & 25.253 & 0 & 19.9 & 7.18 & 10.67 & 2.68 & 3.42 & 0.35 \\
\hline DN87 & Phase n.5 & & 5.2 & 27.25 & 21.397 & 0 & 22.6 & 2.53 & 12.67 & 2.83 & 4.01 & 0.49 \\
\hline DN90 & Phase n.5 & & 5.2 & 26.32 & 26.04 & 0 & 22.6 & 2.53 & 12.67 & 2.83 & 4.01 & 0.49 \\
\hline
\end{tabular}


(c)

\begin{tabular}{|c|c|c|c|c|c|c|c|c|c|c|c|c|c|c|}
\hline \multicolumn{15}{|c|}{ Representative analyses of trace elements (ppm) of Jabal al Haruj al Aswad basaltic rocks } \\
\hline & Intrusion & Sc & $\mathrm{V}$ & $\mathrm{Cr}$ & Co & $\mathrm{Ni}$ & $\mathrm{Cu}$ & $\mathrm{Zn}$ & $\mathrm{Rb}$ & $\mathrm{Sr}$ & $\mathrm{Y}$ & $\mathrm{Zr}$ & $\mathrm{Nb}$ & Mo \\
\hline DN19 & Phase 1.1 & & 195.7 & 337.5 & 56.8 & 190.5 & 69.2 & 70.6 & 4.9 & 332.8 & $<1$ & 86.8 & 10.1 & 1.6 \\
\hline DN49 & Phase 1.1 & & 227.4 & 319.9 & 52.9 & 120.4 & 48.0 & 70.2 & 5.2 & 179.2 & $<1$ & 72.9 & 10.0 & 2.4 \\
\hline DN50 & Phase 1.1 & & 203.6 & 295.6 & 51.7 & 134.8 & 47.1 & 63.5 & 6.7 & 179.9 & $<1$ & 70.4 & 9.7 & 2.1 \\
\hline DN52 & Phase 1.1 & & 207.5 & 281.4 & 50.7 & 116.9 & 54.4 & 59.6 & 4.2 & 184.0 & $<1$ & 66.1 & 9.2 & 2.0 \\
\hline DN53 & Phase 1.1 & & 199.1 & 277.3 & 58.6 & 117.6 & 34.4 & 61.6 & 4.4 & 174.5 & $<1$ & 64.0 & 7.8 & 2.3 \\
\hline DN54 & Phase 1.1 & & 201.6 & 281.1 & 53.9 & 160.5 & 73.0 & 63.8 & 6.3 & 197.3 & $<1$ & 74.4 & 8.7 & 1.7 \\
\hline DN97 & Phase 1.1 & & 205.7 & 292.1 & 47.2 & 186.6 & 68.1 & 61.0 & 6.4 & 220.1 & $<1$ & 81.0 & 11.0 & 2.7 \\
\hline DN64 & Phase 1.1 & & 206.9 & 322.1 & 51.1 & 184.9 & 53.4 & 62.6 & 5.5 & 306.2 & $<1$ & 88.1 & 10.7 & 2.3 \\
\hline DN5 & Phase 1.1 & & 211 & 289.0 & 59.6 & 150.4 & 47.2 & 66.3 & 5.8 & 198.9 & $<1$ & 76.5 & 11.1 & 2.5 \\
\hline DN12 & Phase 1.1 & & 196.5 & 273.4 & 54.6 & 141.5 & 46.0 & 66.0 & 5.1 & 238.5 & $<1$ & 79.8 & 10.8 & 2.4 \\
\hline DN13 & Phase 1.1 & & 196.8 & 287.4 & 51.2 & 134.1 & 41.3 & 62.3 & 5.3 & 221.6 & $<1$ & 71.0 & 9.5 & 1.7 \\
\hline DN17 & Phase 1.1 & & 204.7 & 325.3 & 58.0 & 124.6 & 47.0 & 63.8 & 5.4 & 318.8 & $<1$ & 84.8 & 9.1 & 2.8 \\
\hline DN23 & Phase n.2 & & 193.2 & 267.8 & 53.5 & 118.6 & 41.5 & 52.5 & 5.2 & 240.5 & $<1$ & 80.5 & 11.2 & 2.0 \\
\hline DN80 & Phase n.2 & & 210.3 & 305.3 & 54.2 & 136.9 & 68.5 & 70.0 & 5.6 & 206.1 & $<1$ & 83.2 & 11.9 & 1.5 \\
\hline DN94 & Phase n.2 & & 219.2 & 292.7 & 51.7 & 141.6 & 45.5 & 59.7 & 5.4 & 266.2 & $<1$ & 82.8 & 10.7 & 1.8 \\
\hline DN95 & Phase n.2 & & 196.7 & 238.7 & 42.6 & 81.9 & 52.9 & 58.7 & 3.4 & 176.9 & $<1$ & 59.5 & 7.2 & 1.8 \\
\hline DN35 & Phase n.3 & & 197.3 & 339.1 & 53.5 & 193.1 & 55.0 & 66.8 & 5.6 & 306.4 & $<1$ & 84.2 & 8.6 & 1.6 \\
\hline DN43 & Phase n.3 & & 208.3 & 329.4 & 47.1 & 114.0 & 60.2 & 62.2 & 4.9 & 405.6 & $<1$ & 99.3 & 12.4 & 1.5 \\
\hline DN51 & Phase n.3 & & 186.1 & 265.0 & 45.2 & 124.2 & 48.1 & 56.9 & 4.6 & 247.0 & $<1$ & 71.0 & 9.0 & 1.9 \\
\hline DN55 & Phase n.3 & & 201.2 & 282.1 & 43.8 & 137.3 & 50.5 & 60.0 & 5.6 & 235.9 & $<1$ & 80.4 & 10.4 & 2.0 \\
\hline DN68 & Phase n.3 & & 228.1 & 316.0 & 61.2 & 153.8 & 47.7 & 65.0 & 5.2 & 388.3 & $<1$ & 101.4 & 12.4 & 1.9 \\
\hline DN30 & Phase n.4 & & 217.1 & 268.5 & 48.8 & 108.3 & 32.6 & 62.5 & 7.1 & 186.6 & $<1$ & 76.6 & 9.8 & 1.9 \\
\hline DN33 & Phase n.4 & & 211.9 & 304.1 & 53.4 & 114.7 & 44.5 & 74.7 & 6.0 & 183.1 & $<1$ & 68.9 & 8.3 & 1.5 \\
\hline DN69 & Phase n.4 & & 211.2 & 283.3 & 46.5 & 103.0 & 35.2 & 58.9 & 5.8 & 187.9 & $<1$ & 71.5 & 10.4 & 2.1 \\
\hline DN92 & Phase n.4 & & 237.1 & 358.4 & 56.9 & 133.6 & 47.9 & 70.7 & 6.3 & 176.9 & $<1$ & 75.4 & 9.8 & 2.6 \\
\hline DN108 & Phase n.4 & & 215.0 & 345.2 & 56.7 & 172.8 & 49.4 & 71.7 & 4.7 & 202.9 & $<1$ & 75.1 & 10.7 & 1.6 \\
\hline DN86 & Phase n.5 & & 243.2 & 327.6 & 57.3 & 139.1 & 52.4 & 68.8 & 6.1 & 200.1 & $<1$ & 75.1 & 9.6 & 1.0 \\
\hline DN87 & Phase n.5 & & 260.0 & 361.3 & 60.1 & 165.3 & 59.1 & 70.8 & 7.0 & 236.6 & $<1$ & 91.6 & 13.9 & 1.7 \\
\hline DN90 & Phase n.5 & & 229.1 & 300.1 & 57.1 & 130.6 & 54.3 & 76.8 & 6.1 & 178.5 & $<1$ & 71.0 & 9.2 & 2.4 \\
\hline
\end{tabular}


(d)

\begin{tabular}{|c|c|c|c|c|c|c|c|c|c|}
\hline \multicolumn{10}{|c|}{ Representative analyses of trace elements (ppm) continued } \\
\hline & Intrusion & Sn & $\mathrm{Ba}$ & $\mathrm{La}$ & $\mathrm{Yb}$ & $\mathrm{Hf}$ & Та & $\mathrm{Pb}$ & Th \\
\hline DN19 & Phase 1.1 & 3.2 & 333.4 & 16.5 & 4.4 & 3.7 & 3.7 & 3.9 & $<1$ \\
\hline DN49 & Phase 1.1 & 5.9 & 176.6 & 6.0 & 4.1 & 2.8 & 4.2 & 3.9 & $<1$ \\
\hline DN50 & Phase 1.1 & 2.2 & 177.5 & 17.4 & 4.2 & 3.7 & 3.7 & 4.7 & 2.3 \\
\hline DN52 & Phase 1.1 & 2 & 191.6 & 13.5 & 3.9 & 1.8 & 4.0 & 4.8 & 4.4 \\
\hline DN53 & Phase 1.1 & 2.4 & 166.4 & 15.0 & 4.1 & 3.4 & 4.0 & 4.1 & $<1$ \\
\hline DN54 & Phase 1.1 & 2.4 & 241.3 & 11.4 & 4.2 & 3.0 & 3.5 & 7.7 & $<1$ \\
\hline DN97 & Phase 1.1 & 3.2 & 194.7 & 13.7 & 4.3 & 3.4 & 4.0 & $<1$ & $<1$ \\
\hline DN64 & Phase 1.1 & 2.4 & 616.0 & 7.1 & 4.1 & 2.2 & 3.6 & 4.8 & 3.0 \\
\hline DN5 & Phase 1.1 & 0.6 & 203.6 & 19.0 & 4.2 & 3.4 & 5 & 6.4 & 4.0 \\
\hline DN12 & Phase 1.1 & 3.7 & 930.6 & 15.3 & 4.2 & 4.6 & 3.6 & 4.3 & 2.0 \\
\hline DN13 & Phase 1.1 & 2.9 & 157.7 & 17.9 & 4.2 & 3.5 & 3.8 & 4.4 & $<1$ \\
\hline DN17 & Phase 1.1 & 2.5 & 214.2 & 15.5 & 4.1 & 2.2 & 3.4 & 4.5 & 1.0 \\
\hline DN23 & Phase n.2 & 1.9 & 194.3 & 10.2 & 4 & 2.8 & 3.6 & 2.8 & 2.2 \\
\hline DN80 & Phase n.2 & 3.4 & 189.8 & 12.3 & 4 & 3.1 & 3.6 & 5.5 & 0.8 \\
\hline DN94 & Phase n.2 & 2.0 & 1070 & 13.4 & 4 & 4.9 & 2.8 & 2.2 & 2.1 \\
\hline DN95 & Phase n.2 & 4.3 & 164.1 & 7.2 & 3.9 & 1.4 & 3.2 & 4.9 & 2.8 \\
\hline DN35 & Phase n.3 & 2.4 & 1062 & 15.7 & 4.1 & 4.4 & 3.3 & 6.3 & 1.0 \\
\hline DN43 & Phase n.3 & 1.5 & 764.0 & 15.4 & 4 & 3.4 & 4.1 & 3.8 & 2.3 \\
\hline DN51 & Phase n.3 & 2.5 & 310.3 & 10.5 & 3.9 & 3.5 & 3.2 & 6.4 & $<1$ \\
\hline DN55 & Phase n.3 & 2.2 & 321.3 & 12.3 & 4 & 2.3 & 3.1 & 5.0 & 2.1 \\
\hline DN68 & Phase n.3 & 3.8 & 663.0 & 17.0 & 4.1 & 3.8 & 3.3 & 3.7 & $<1$ \\
\hline DN30 & Phase n.4 & 3.7 & 219.8 & 12.6 & 3.9 & 3.5 & 3.6 & 5.5 & 3.0 \\
\hline DN33 & Phase n.4 & 4.1 & 352.8 & 16.4 & 4.1 & 1.5 & 4.6 & 4.9 & $<1$ \\
\hline DN69 & Phase $n .4$ & 3.5 & 199.4 & 15.6 & 4.1 & 3.3 & 4 & 6.7 & 1.4 \\
\hline DN92 & Phase n.4 & 2.1 & 176.8 & 19.5 & 4.1 & 4 & 4 & 4.6 & $<1$ \\
\hline DN108 & Phase n.4 & 2.5 & 292.0 & 11.3 & 4.2 & 4.1 & 3.9 & 5.9 & 1.0 \\
\hline DN86 & Phase n.5 & 1.1 & 180.9 & 14.7 & 4.2 & 3.4 & 4 & 4.3 & 2.0 \\
\hline DN87 & Phase n.5 & 2.4 & 182.0 & 29.9 & 4.2 & 3.3 & 3.4 & 6.4 & 2.5 \\
\hline DN90 & Phase n.5 & 2.8 & 173.0 & 22.9 & 4.1 & 2.5 & 3.5 & 6.1 & 2.0 \\
\hline
\end{tabular}

The chemical composition ranges of the investigated samples as a whole are relatively narrow within each individual basaltic phase and also within the different phases that existed there. The chemical ranges of the studied samples are somewhat restricted.

According to the CIPW norm, the analysed rocks are characterized by a high normative olivine content, with at most a low content of normative nepheline. Hence, these rocks can be classified as olivine basalt. The occurrence of normative hypersthene leads to rock being classified as normal olivine transitional basalt. $\mathrm{SiO}_{2} \mathrm{ranges}$ from 49.5\% to 52.7\% together with $\mathrm{Mg \#} \mathrm{(Mg/(Mg}+$ Fet)) values of 52 to 62 indicate that the studied basaltic rocks are slightly to moderately evolved (less primitive). Thus, they are not considered as the primary melt of 
the primitive mantle. The studied basaltic rocks are actually basalt on the basis of their silica content.

The petrographical criteria obtained from the studied basaltic rocks are not strictly conclusive in their classification. However, the modal contents cannot be accurately determined in most cases in volcanic rocks because of the fine grain size and presence of glassy materials. Therefore, some chemical criteria should be used to determine the basalt type of the studied rocks. The chemical classifications use parameters such as the existence of oxides, functions of oxides and ratios. Various suggestions have been proposed in this domain by different authors.

The volcanic rocks may be subdivided into two members of the major magma series, alkalic and subalkalic; however, although the boundary between them is marked as a solid line, it is actually transitional.

From the various literature dealing with petrochemical classification, it is clear that four types of basaltic rocks can be distinguished: tholeiitic basalts, transitional basalts, alkali basalts and high alumina basalts or calc-alkaline basalts, Cox et al. [19]. The concept of transitional basalts between the tholeiitic and alkali basalt type has been clarified by many authors, e.g. Middlemost, [20]. For the chemical classification of the basaltic rocks under consideration variation diagrams are employed using major elements, which are introduced in the following.

According to Middlemost [21] the investigated samples are transitional basalts of either a soda or potash series. This can be accomplished by an empirical boundary line on a soda vs potash diagram. The studied transitional basalts were found to belong to the soda series (Figure 7).

In addition to the classifications based on major elements, certain variation diagrams have been suggested by Winchester and Floyd [23] to classify the basaltic rocks using five minor and trace elements (Ti, P, Zr, YNb) to produce a set of binary diagrams that discriminate between tholeiitic and alkali basalts (Figure 8).

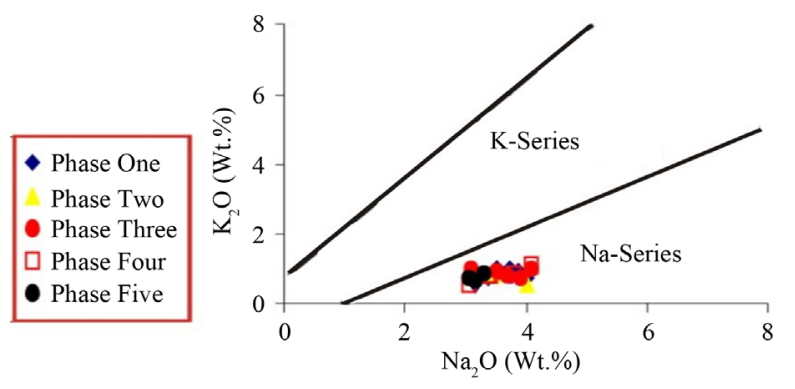

Figure 7. $\mathrm{K}_{2} \mathrm{O}$ versus $\mathrm{Na}_{2} \mathrm{O}$ (wt\%) diagram, showing the subdivision of the transitional magma series into the Na sub-series of the samples in question, after Winter [22].
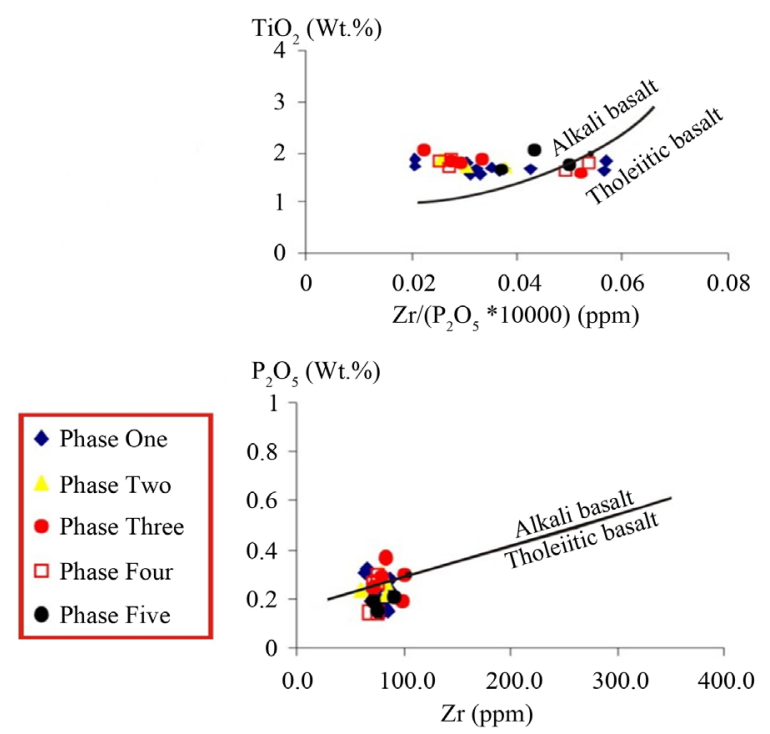

Figure 8. Minor $\left(\mathrm{TiO}_{2}, \mathrm{P}_{2} \mathrm{O}_{5}\right)$ and trace $(\mathrm{Zr})$ element variation plots for the classification of the studied rocks as alkalic and tholeiitic basalts, after Winchester and Floyd [23]. 
The $\mathrm{P}_{2} \mathrm{O}_{5}$-Zr diagram shows that the investigated samples are plotted close to the boundary of the alkali between the alkali basalt and tholeiitic basalt, whereas the $\mathrm{TiO}_{2}-\mathrm{Zr} / \mathrm{P}_{2} \mathrm{O}_{5}$ diagram shows that most of samples are situated in alkali field (Figure 8). This is expected since these diagrams use phosphorous as a principal component, which shows varying degrees of mobility, as stated by Rollinson [24]. Moreover, the $\mathrm{Zr}$ values of the studied samples are generally low.

The major element data contains only $\mathrm{Fe}_{2} \mathrm{O}_{3}$ as total iron. To obtain appropriate CIPW norms these values had to be recalculated into $\mathrm{Fe}_{2} \mathrm{O}_{3}$ and $\mathrm{FeO}$. In the current study the ratio of $\mathrm{Fe}_{2} \mathrm{O}_{3} / \mathrm{FeO}=0.2$ was used (Less et al. [12]; Peregi et al., [5]). Middlemost [20] has proposed a range of oxidation ratios $\left(\mathrm{Fe}_{2} \mathrm{O}_{3} / \mathrm{FeO}\right)$ for use with volcanic rocks drawn from geological literature; that of basalts is $\mathrm{Fe}_{2} \mathrm{O}_{3} / \mathrm{FeO}=0.2$, Rollinson, [24].

There are various published programs for the calculation of the CIPW. The CIPW normative calculation in the current study was carried out by Norm4 software, written by Kurt Hollocher of the Geology Department, Union College, New York State. Several methods for the normative classification of basaltic rocks have been proposed. Yoder and Tilley [25] devised a scheme for classifying basaltic rocks by their CIPW norms using a basalt tetrahedron. Several modifications have been suggested to this classification and various schemes have been proposed by later investigators. Among these, Thompson [26] proposed a classification scheme for basalts based upon CIPW normative proportions of Ne, Ol, Di, Hy and Q. The three equilateral triangles of Thompson's diagram [26] represent basaltic and related rocks, which are respectively undersaturated, saturated and oversaturated: silica-undersaturated basalts (alkali basalts) are characterized by normative $\mathrm{Ol}+\mathrm{Ne}$; silica-saturated basalts (olivine tholeiite) are characterized by $\mathrm{Hy}+\mathrm{Ol}$; and, silica-oversaturated basalts (quartz tholeiites) are characterized by normative Q + Hy. With regard to normative minerals, the most studied samples are classified into hypersthene-normative; Wilkinson [27] considered hypersthene-normative rocks to be transitional or mild alkaline.

Busrewil and Oun [28] suggested that the transitional basalt is applied to basaltic rock that exhibits a nepheline-free norm. Three samples belonging to Phase 1, two samples from Phase 2 and two samples from Phase 3 were classified into nepheline-normative alkaline basalts (ne $=0.10-4.8$; alkali-ol basalts), and one sample from Phase 4 was located at the boundary between ol-tholeiite and quartz tholeiite; this latter sample is near to oversaturation in $\mathrm{SiO}_{2}$, which is a common feature of tholeiitic basalts (Figure 9).

The two main types of variation diagrams currently used are bivariate plots and triangular diagrams. A bivariate plot shows the variation between samples and identifies trends. Elements plotted along the $\mathrm{x}$-axis of the diagram should be selected either to show maximum variability between samples or illustrate a particular geochemical process. Normally, in basic igneous rock MgO might be selected, Rollinson, [24].

One of the most commonly used alternatives to the Harker diagram is MgO plot. This is most appropriate for a rock series which includes abundant mafic members, as in this case the range of $\mathrm{SiO}_{2}$ concentrations may be small. The changes in selected major and minor element oxides are plotted against $\mathrm{MgO}$ for representative sam-

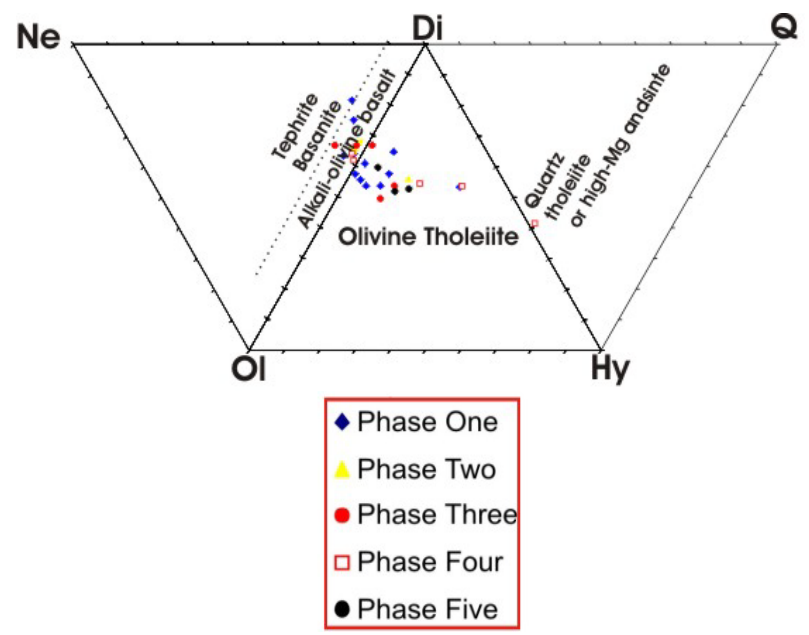

Figure 9. The classification of the studied samples according to their CIPW normative composition expressed as Ne-Ol-Di, OlDi-Hy, Di-Hy-Q (basalt tetrahedron, after Thompson, [26]). 
ples from the study area. The analyses exhibit an evolutionary trend representing slight increases in $\mathrm{SiO}_{2}, \mathrm{~K}_{2} \mathrm{O}$ and $\mathrm{Na}_{2} \mathrm{O}$, along with decrease $\mathrm{CaO}$, FeOt and MgO. Clearly, $\mathrm{MgO}$ increases with increased FeOt. However, in spite of this overall trend there is no consistent chemical pattern related to each phase.

Vague correlations between $\mathrm{MgO}$ and most other oxides in the studied samples probably suggest that these are related by different degrees of partial melting rather than by fractional crystallization. The relatively low MgO of less than $7.5 \mathrm{wt} \%$ and $\mathrm{Ni}(81-193 \mathrm{ppm})$ and $\mathrm{Cr}(238$ - $361 \mathrm{ppm})$ contents indicates that the studied basaltic rocks exhibit slight to moderate fractionation of olivine and/or spinel. Consequently, the basaltic rocks are not primary magmas.

On average in the studied samples, the lava flows of Phase 5 and two samples from Phase 3 are more primitive. $\mathrm{MgO}$ is an important component of the solid phases in equilibrium with mafic melts and shows a great deal of variation, either as a consequence of the breakdown of magnesium phases during partial melting or as a result of their removal during fractional crystallization. Below 7\% MgO-CaO correlates positively with MgO, indicating the removal of $\mathrm{CaO}-\mathrm{MgO}$ from the liquid due to the crystallization of plagioclase and clinopyroxene. However, above 7\% $\mathrm{MgO}$ and $\mathrm{CaO}-\mathrm{MgO}$ correlate negatively, because this part of the trend is controlled entirely by the olivine. Inflections are most obvious where the number of fractionating minerals is small, Rollinson, [24].

Trace element data provide a clearer view than the major and minor elements of distinct sources for the various magma types. In the current study the focus is on the trace elements, which due to enrichment and depletion are revealing about the nature of the basalt plateau. The trace element data can be used to evaluate the source composition, degree of partial melting and subsequent processes. The ratios of incompatible elements have been employed to distinguish between source reservoirs. The concentrations of the compatible trace elements $(\mathrm{Ni}, \mathrm{Cr}$, $\mathrm{Co}, \mathrm{Cu}$ ) are low in the studied samples, which supports our contention that they are not primary magma.

A characteristics feature of the studied volcanic rocks is their relatively constant ratios of certain incompatible trace elements ( $\mathrm{Nb} / \mathrm{Zr}$ and $\mathrm{Rb} / \mathrm{Zr}$ ) (Table 4). Relative constancy of ratios of incompatible elements provides strong evidence of a common source (homogenous). Also, the $(\mathrm{Zr} / \mathrm{Nb})$ ratios of studied basaltic rocks are less than 10, which indicates an ocean island basalt (OIB) source, Winter [22].

Crustal contamination would certainly alter the ratios of $\mathrm{Zr} / \mathrm{Nb}$ and $\mathrm{Hf} / \mathrm{Ta}$. The studied mafic samples have similar ratios, so the effect of crustal contamination on the studied basaltic rocks is probably minor, Baker [29].

Several variants of the spider diagram have been used in the literature, with different elements and different normalization schemes. The order of the elements along the abscissa may vary slightly between different authors. Spider diagrams are flexible, and a variety of elements and normalizations have been displayed using them, Winter, [22]. In the current study, the comparison of the geochemical composition of the different rock samples, representative of different phases, with the MORB-normalized trace element patterns was used by Pearce [30].

Figure 10 compares the incompatible elements of each sample with the most abundant igneous rocks on the planet. The large ionic lithophile (LIL) elements are on the left-hand side of the diagram and the high field strength (HFS) elements are on the right. Both are arranged in order of increasing incompatibility away from the margins, so that the most incompatible elements are just left of the centre of the diagram, Winter [22].

The MORB-normalized trace element patterns of representative samples of different volcanic phases are given in Figure 10. Based on these diagrams, the patterns show a fairly close similarity in the shape and concentration range. The diagrams show a slightly different concentration of some incompatible trace elements

Table 4. Ratios between some trace elements of the studied mafic rocks that could be used for their petrogenesis.

\begin{tabular}{|c|c|c|c|c|c|c|c|c|c|c|c|c|c|c|}
\hline \multicolumn{13}{|c|}{ Phase One } & \multicolumn{2}{|c|}{ Phase Two } \\
\hline Sample No. & 97 & 19 & 12 & 13 & 17 & 5 & 49 & 50 & 52 & 53 & 54 & 64 & 23 & 80 \\
\hline $\mathrm{Nb} / \mathrm{Zr}$ & 0.135 & 0.116 & 0.135 & 0.134 & 0.106 & 0.144 & 0.136 & 0.137 & 0.139 & 0.121 & 0.117 & 0.120 & 0.138 & 0.143 \\
\hline \multirow[t]{2}{*}{$\mathrm{Rb} / \mathrm{Zr}$} & 0.078 & 0.056 & 0.063 & 0.074 & 0.063 & 0.075 & 0.070 & 0.095 & 0.069 & 0.084 & 0.062 & 0.064 & 0.067 & 0.065 \\
\hline & \multicolumn{3}{|c|}{ Phase Two } & \multicolumn{3}{|c|}{ Phase Three } & \multicolumn{5}{|c|}{ Phase Four } & \multicolumn{3}{|c|}{ Phase Five } \\
\hline Sample No. & 94 & 95 & 35 & 43 & 51 & 55 & 30 & 33 & 69 & 92 & 108 & 86 & 90 & 87 \\
\hline $\mathrm{Nb} / \mathrm{Zr}$ & 0.129 & 0.120 & 0.125 & 0.126 & 0.128 & 0.122 & 0.127 & 0.120 & 0.145 & 0.129 & 0.142 & 0.127 & 0.128 & 0.151 \\
\hline $\mathrm{Rb} / \mathrm{Zr}$ & 0.065 & 0.056 & 0.066 & 0.048 & 0.065 & 0.051 & 0.092 & 0.086 & 0.081 & 0.083 & 0.062 & 0.081 & 0.085 & 0.075 \\
\hline
\end{tabular}



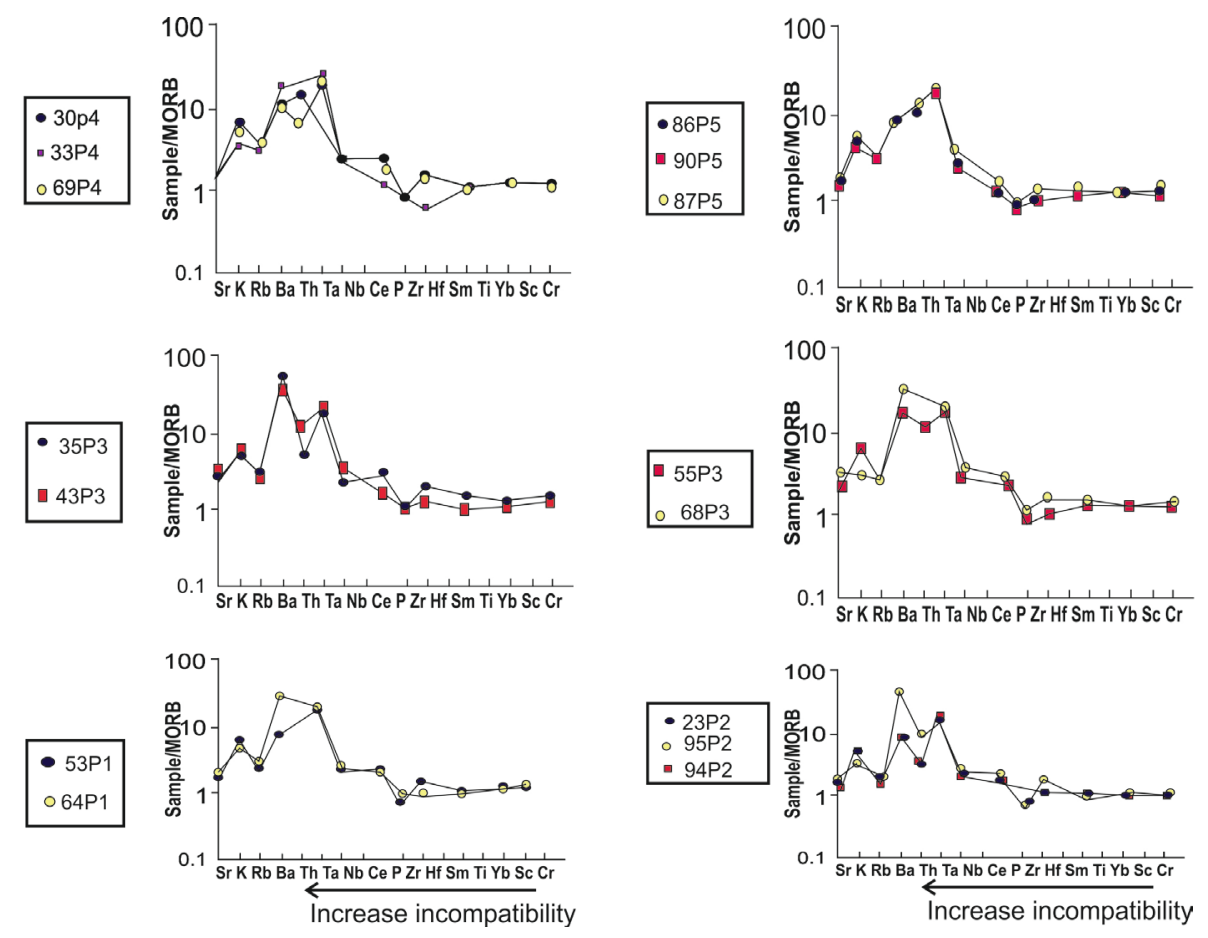

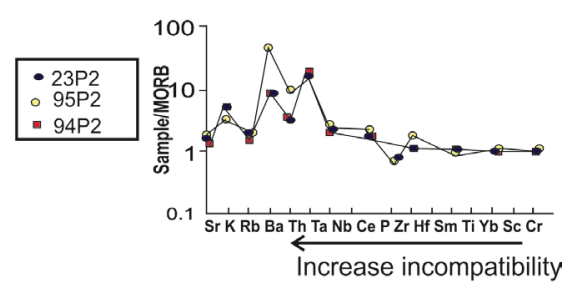

Figure 10. MORB-normalized patterns for selected samples from the study area, representative of different phases from Phases 1 to 5 (normalized data is greater than one). The Ba concentration of the studied samples shows extreme variation in some samples due to very high sensitivity to secondary processes.

within samples of the same phase, and also between samples representative of different phases, but they showed nearly identical behaviour for the less incompatible trace elements (Hf, Sm, Ti, Yb).

The studied basaltic rocks showed more peaks and troughs, reflecting the variable behaviour of the elements involved. Basalts from the study area are clearly enriched by the whole spectrum of more incompatible elements relative to MORB samples (normalized values greater than one). They show a broad central hump in which both LIL (K, Ba), and HFS (Ta) elements also reveal slight negative anomalies in Rb, Nb and Zr. The rocks display similar patterns of the peaks and troughs, which strongly suggests they share a common parent and subsequent processes, Winter [22]. Thus, based on this, the basalts could have a common genesis.

The basaltic samples from different volcanic phases show a fairly similar character to each other in their geochemical behaviour. Consequently, one phase cannot be distinguished from another based on their trace element contents.

From the consistent negative slopes, it can be deduced that the slope of the studied basaltic rocks are distinct from MORB slope and appear to originate in the lower enriched mantle reservoir, although a very low degree of partial melting may also produce LREE-enriched melts from primordial or slightly depleted sources, Cox et al. [19]. The pattern is regarded as typical of melts generated from relatively undepleted mantle material.

Spikes at LIL (Rb, Ba, K) may reflect enrichment, perhaps due to contamination of magma by continental crust. Negative $\mathrm{Nb}$ anomalies are also characteristic of the continental crust and may be an indicator of crustal involvement in magma processes, Rollinson [24]. Enriched LIL and the slight alkaline of the studied basaltic rocks suggest derivation from an enriched mantle source, Aboazom [31].

The high LIL/HFS pattern and negative $\mathrm{Nb}$ anomaly is a feature of many intracontinental volcanics, suggesting this pattern may be also a feature of the subcontinental lithospheric mantle (Fitton et al. [32]; Hawesworth [33]).

As the degree of partial melting increases, the proportion of a more incompatible element with respect to a less incompatible element decreases as it is progressively diluted, so that (as illustrated by Figure 11) the La/Nb ratio should decrease and the $\mathrm{La} / \mathrm{Zr}$ ratio should increase. The extent of partial melting should thus increase from the upper left to the lower part of the mantle partial melting curve, Winter [22]. 


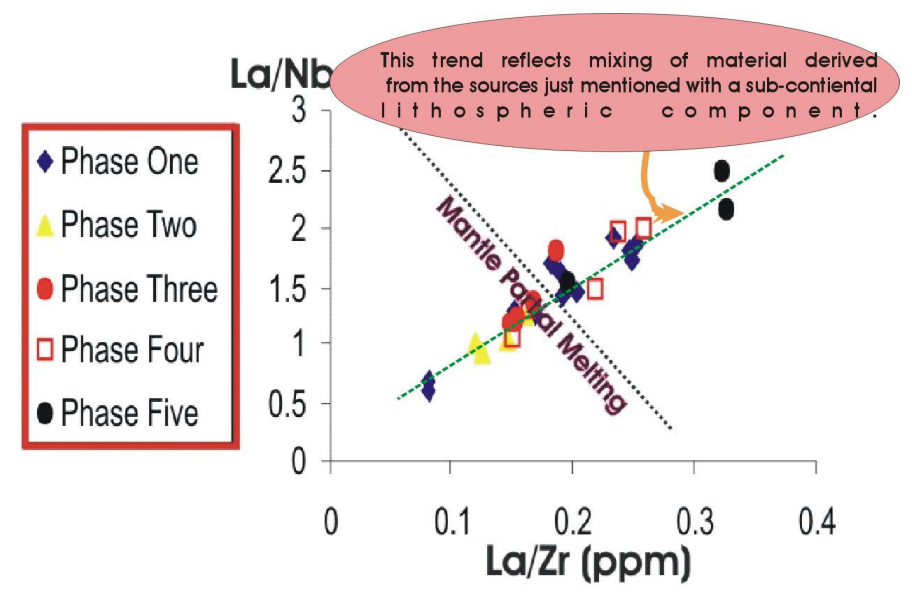

Figure 11. La/Zr vs La/Nb for the studied basalt samples, after Hopper et al. [34].

The distinct phases suggest a single source and probably similar degrees of partial melting. The positive trend in Figure 12 reflects the mixing of material derived from the source, Hopper et al. [34].

The elements of the first transition series (Ti, V, Cr, Mn, Fe, Co, Ni, Cu, Zn) vary in geochemical behaviour, and most of them are considered to be compatible elements. Transitional element plots have been mainly used with basalts as a means of exploring the geochemical behaviour of the first transitional elements, Rollinson [24].

Figure 12 shows consistent trends for most studied samples representative of different phases on chondritenormalized plots. They show progressive depletion from $\mathrm{Ti}$ to $\mathrm{Ni}$ and have a positive Ti anomaly and negative $\mathrm{Cr}$ anomaly. $\mathrm{Cu}$ and $\mathrm{Zn}$ are more variable and their concentrations may not reflect the original igneous values, (Longmuir et al. [35]. Anomalies in $\mathrm{Ni}$ and $\mathrm{Cr}$ concentrations may reflect the role of olivine (Ni) and clinopyroxene or spinel $(\mathrm{Cr})$; $\mathrm{Ti}$ anomalies indicate the role of $\mathrm{Fe}$-Ti oxides as opaque minerals.

Despite differences in the major element chemistry, the pronounced and very close patterns of similarity of trace elements of all the samples representative of the different phases certainly suggest there is a common source for the mantle peridotite beneath Al-Haruj province.

\subsection{Comparison of the Studied Mafic Rocks with the Mid-Oceanic Ridge (OIB)}

A broad spectrum of trace elements for the studied basalts rock can be compared using composite (average) OIB, as proposed by Sun [37] (Figure 13). The patterns of the studied basaltic rocks are similar in the shape to OIB, but vary in the concentration range of some incompatible trace elements. OIB enrichment in LIL ( $\mathrm{Sr}, \mathrm{K}, \mathrm{Rb}$ ) thus suggests a somewhat depleted mantle source relative to OIB, Winter [22].

\subsection{The Results of Correspondence Analysis}

There is a large body of literature on the statistical treatment of geochemical data. Correspondence analysis is a descriptive and exploratory technique designed to analyse multi-way tables containing some measures of correspondence between rows (observations) and columns (variables). The results provide information that is similar in concept to those produced by correlation techniques. The term "inertia" in correspondence analysis can be thought of as a measurement of variation in data.

Obviously, the final goal of correspondence analysis is to find a graphical interpretation (meaning) from extracted dimensions (dimension 1 on the $\mathrm{x}$-axis and dimension 2 on the $\mathrm{y}$-axis). Thus, one can make the interpretation that the great similarity between the average of the elements of each phase with regard to the correlation between most HFS, LIL and transitional metal elements is more in Phase 4. However, as has been indicated, it is appropriate to make general statements about the nature of the dimensions (Figure 14).

The variance is explained by coordinates (dimensions) of more than $98 \%$ (96.03\% of inertia on dimension 1 and $2.192 \%$ of inertia on dimension 2), which implies the relation between the average of the different phases and relation of elements between them and the relation between the elements and average of the five phases are highly significant, and so it can be stated with great confidence. 

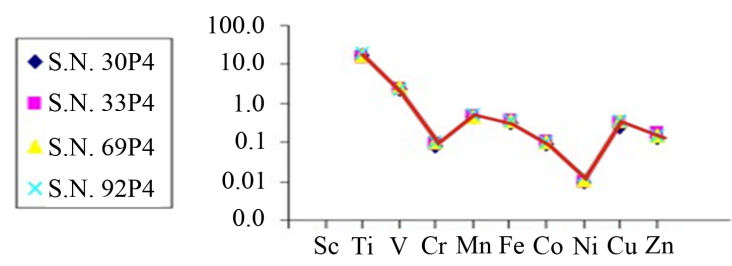

Sc Ti V Cr Mn Fe Co Ni Cu Zn
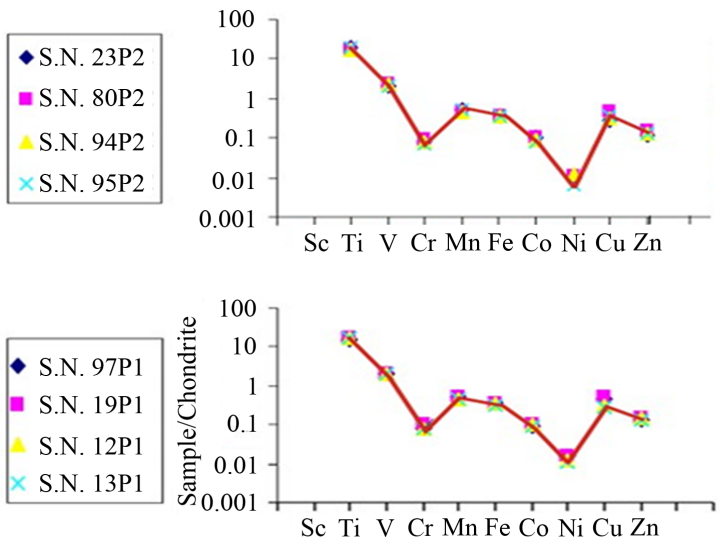
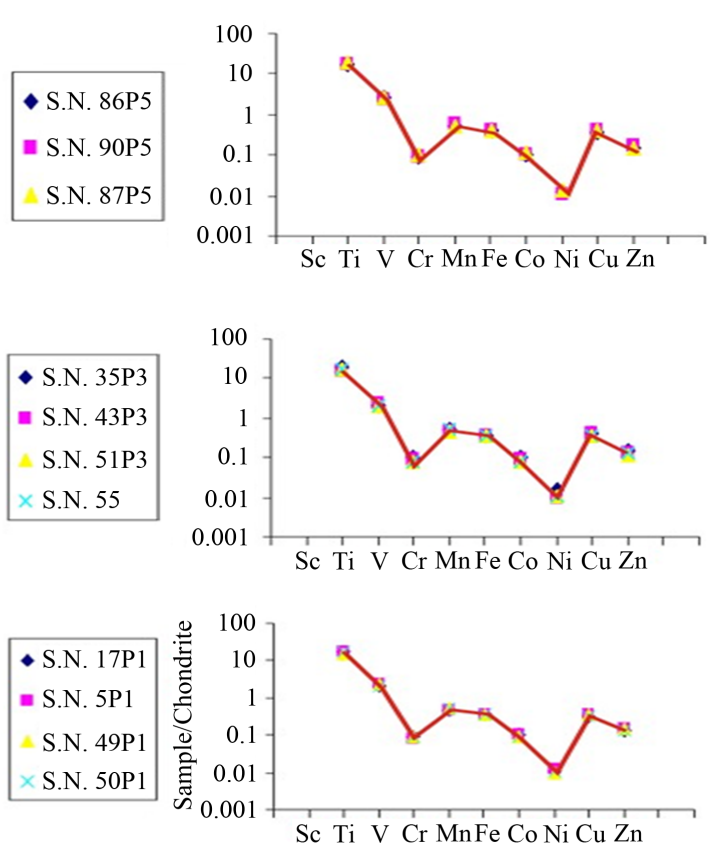

Figure 12. Transitional metal concentrations in most studied samples representative of the five phases, normalized to chondritic values, showing the same behaviour of transitional metal of different phases. The normalized values are taken from Kay and Hubbard [36].

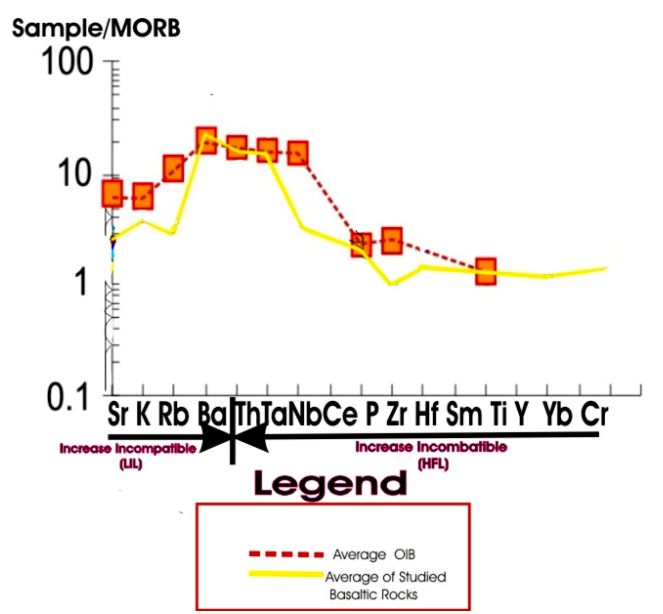

Figure 13. MORB-normalized spider diagram for average OIB, data from Rollinson, [24] and average representative analyses of the studied samples.

Judging the quality of the average of five phases expresses the value and importance of each phase in the current study, so in Table 5 it can be clearly observed that all phases are of highly significant importance. The correspondence analysis is useful for drawing attention to the likelihood that all phases were generated by sharing a common source.

\subsection{Tectonic Environments}

The approach of using geochemical analyses to try to discriminate magmas into different tectonic settings is described by several authors, and is probably best attributed to Pearce ([38] [39]), Pearce and Cann ([40] [41]), Pearce et al. ([42] [43]), Pearce and Norry [44] and Wood et al. [45]. They accepted that the major, minor and trace elements of basalts should reflect their geotectonic position, because the geochemistry of basalts is dependent on the crustal nature of the area in which they were formed. 


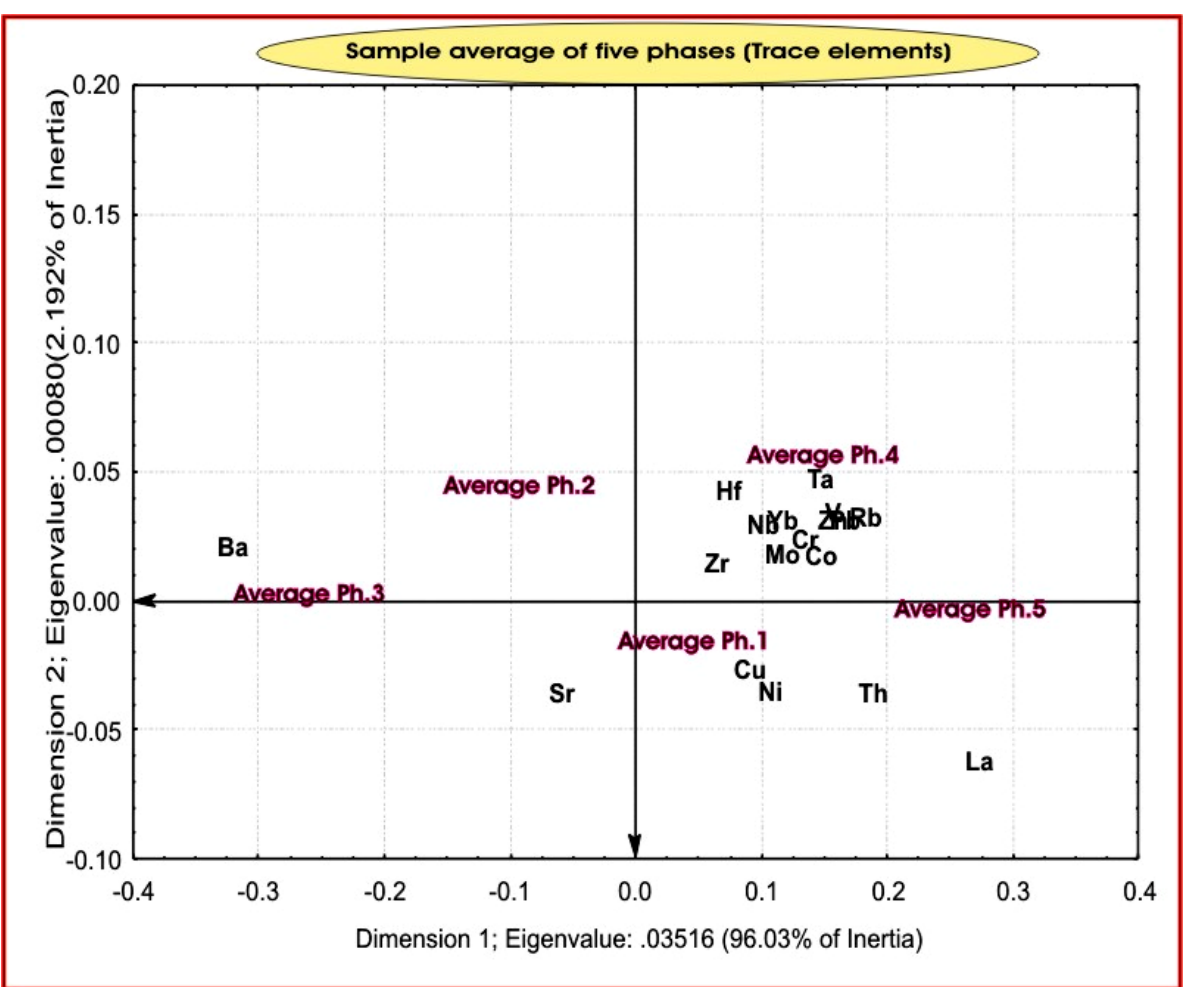

Figure 14. Correspondence analysis for trace elements of the average five phases presented in the area investigated, plotted on coordinates (on dimension 1 and dimension 2).

Table 5. The results of correspondence analysis for the average of the five phases.

\begin{tabular}{ccccc}
\hline Aver. Phases & Colum & Coordinate & Coordinate & Quality \\
\hline Average Ph. 1 & 1 & 0.052836 & -0.032650 & 0.818930 \\
Average Ph. 2 & 2 & -0.093243 & 0.028226 & 0.904941 \\
Average Ph. 3 & 3 & -0.262192 & -0.0125540 & 0.995548 \\
Average Ph. 4 & 4 & 0.135643 & 0.042508 & 0.970742 \\
Average Ph. 5 & 4 & 0.261218 & -0.020566 & 0.991675 \\
\hline
\end{tabular}

Floyd and Winchester [46] found that basalts with similar chemistry may be produced in different tectonic regimes, since their compositions reflect the nature of the particular mantle and the melting processes operating rather than the regime in which they are found. Probably, this often makes the chemical designation of ancient basalt less certain.

Wood et al. [47] considered the effects of crustal contamination which were not considered by Pearce and Cann [41] and yet which are likely to have a significant influence in the appearance of continental flood basalts and may be a contributory factor in their persistence in classification, Rollinson [24]. There are a large number of discrimination diagrams applicable to basalts and basaltic andesites, which use trace elements and minor and major elements to distinguish the different environments of basalt. Of course, the investigator should not rely wholly on any single technique or plot, Winter [22].

Pearce [38] developed a method that classified basalts in term of their tectonic setting on the basis of their major element patterns. He calculated the discriminant function based upon the eight major oxides and presented discriminant diagrams to identify mid-oceanic ridge basalt (MORB), within plate basalt, oceanic island basalt (OIB), continental flood basalt (CFB), calc-alkaline basalts (CAB), island arc tholeiites (IAT) and shoshonites (SB).

Two discriminant functions, F1 and F2, were identified as follows: 


$$
\begin{aligned}
F 1= & +0.0088 \mathrm{SiO}_{2}-0.0774 \mathrm{TiO}_{2}+0.0102 \mathrm{Al}_{2} \mathrm{O}_{3}+0.0066 \mathrm{FeO}-0.0017 \mathrm{MgO} \\
& -0.0143 \mathrm{CaO}-0.0155 \mathrm{Na}_{2} \mathrm{O}+0.0007 \mathrm{~K}_{2} \mathrm{O} \\
F 2= & -0.0130 \mathrm{SiO}_{2}-0.0185 \mathrm{TiO}_{2}-0.0129 \mathrm{Al}_{2} \mathrm{O}_{3}-0.0134 \mathrm{FeO}-0.0300 \mathrm{MgO} \\
& -0.00204 \mathrm{CaO}-0.0481 \mathrm{Na}_{2} \mathrm{O}-0.071 \mathrm{~K}_{2} \mathrm{O}
\end{aligned} .
$$

It is clear that the majority of the samples investigated are plotted in the field of within-plate basalts (Figure 15).

Wood [47] proposed the Th-Hf-Ta ternary discrimination diagram based upon the immobile (HFS) elements. Using this classification (Figure 16(a)), the studied basaltic rocks plot closer to the fields of within-plate basalts. The elements Th, Hf, and Ta existed in very low concentrations in basalts and cannot accurately determined by XRF analysis.

The advantage of using minor elements as discriminants between basalts is that they are readily detected and more accurately determined than trace elements, Rollinson [24].

Pearce et al. [42] found in an empirical study that it was possible to discriminate between oceanic and continental basalts with a single straight boundary line on a $\mathrm{TiO}_{2}-\mathrm{K}_{2} \mathrm{O}-\mathrm{P}_{2} \mathrm{O}$ triangular diagram (Figure 16(b)). The field oceanic basalts included MORB and OIB. The studied basaltic rocks are plotted around the line separating the two fields.

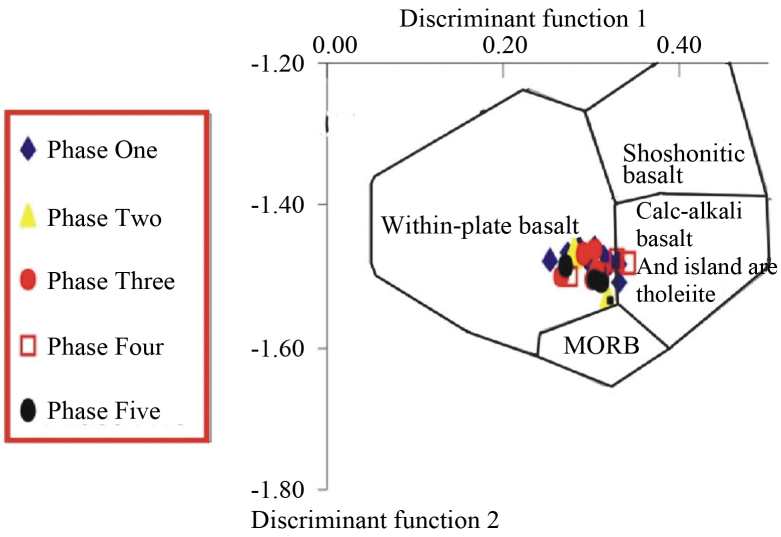

Figure 15. Major element discrimination diagram for basalts, after Pearce [38], showing the most studied samples plot in the field of within-plate basalts.

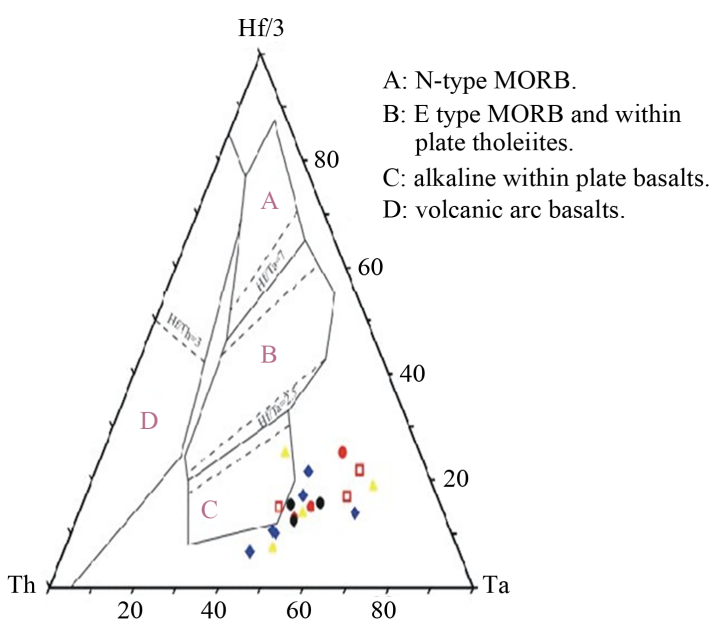

(a)

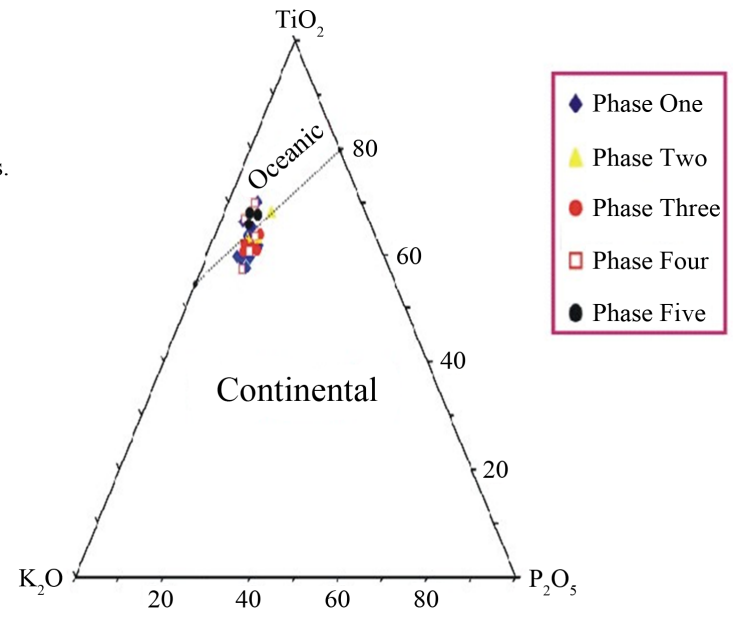

(b)

Figure 16. (a) The Th-Hf/3-Ta ternary discrimination diagram for basalts (after Wood, [47]); the broken lines indicate transitional zones between basalts types. (b) Triangular discrimination diagram between oceanic and continental fields, after Pearce et al. [42]. 


\section{Summary and Discussions}

The tertiary volcanic rocks of Jabal Al Haruj represent the largest volcanic province in Libya covering an area of $\approx 45,000 \mathrm{~km}^{2}$, which originated during several phases of eruptions that were closely associated in space and time.

The study area is located in north-eastern Jabal Al Haruj al Aswad, an area which has not been studied in a thorough manner so far. Nevertheless, the work of Klitzsch [13] gave research details showing the distribution of six major phases and one minor phase. The most important study in this respect was done by Busrewil and Suwesi [2] on the Al Haruj al Aswad. The authors gave a detailed volcanological study of the area and distinguished six volcanic phases.

The division of the volcanic phases used in the present work is based on similar principles to those developed by the earlier authors, which are as follows:

Colour variation of the lava flows of different phases on Landsat TM images.

Degree of weathering of the rocks.

Accessibility of lava fields.

Morphological and lithological characteristics observable in the field.

Superposition of the lava flows and drainage pattern.

Field evidence has revealed that each of these criteria are complementary and cannot be used separately, Busrewil and Suwesi [2]. Field studies included geologic mapping and the identification of mode of occurrence of the volcanics. According to modern age dating, the volcanic activity in the area started in the Late Miocene and lasted until the Late Pleistocene. The first five of the six volcanic phases of the Al Haruj volcanic province are present in the study area. The geological setting, the petrography, the geochemistry and tectonic setting of these occurrences are examined in this study. The following is a summary of the above-mentioned aspects and the main conclusions obtained during the present work.

Field geological mapping was carried out using Landsat TM multispectral satellite imagery. This imagery formed the base of the geological map, which was completed by the addition of topographic contours, locality names and drainage pattern of wadis, as well as other field criteria. The study area is remote, rather inaccessible and extremely difficult terrain. Due to this, the field work was carried out in parallel with the Industrial Research Centre (IRC), Tripoli during their regional geological mapping of SE Libya in March 2006.

The topographic form of the old volcanic phases is highly modified, while the forms of more recent phases are usually fresh and very well preserved. In general, each basalt flow unit begins at the bottom with the most massive part, characterized by a fine to micro-vesicular texture, and it evolves upwards into an obviously vesicular texture. The basalts are dark to medium and pale gray with a porphyritic appearance. A fresh sample was taken from the massive part of each flow unit.

Characteristic features of Phase 1 are the blocky, rough, subangular to subrounded rocky structure and have smooth surfaces, massive and less vesicular. On the satellite image it is distinguished by a bluish colour.

Phase 2 covers a limited geographical extent and outcrops in small scattered localities in parts of the study area. Lava flows from this phase tend to disintegrate into small blocks and slabs, less than $50 \mathrm{~cm}$ in diameter. The vesicles are often elongated in the direction of flow. The presence of lamination is pronounced by the alteration of the variable concentration of vesicles. The accessibility of the area covered by Phase 2 is particularly bad. On the satellite image it is discriminated by a greyish to reddish blue colour, and an almost homogenous surface without any readily recognizable features.

The lava field of Phase 3 covers a large area with subangular blocks that form the rough type of morphology. The blocks are coated by thin dark brown crust providing a rather characteristic field appearance. The fresh broken surface of the rock is black or dark grey. The rocks are readily recognizable on the satellite image by their brown tone or hue, and the hilly morphology dotted by number of large balta (kipukas). The kipuka, or according to their Arabic name balta, are closed shallow depressions within the lava flows surrounded by lava tongues. In Phase 3 the geomorphological dimension of baltas may vary.

The exposures of Phase 4 are widespread in the central Al Haruj al Aswad and in the study area. From the central part of Al Haruj al Aswad large volumes of Phase 4 lava flow poured downward in a more or less radial pattern. The flows are distinguished by their morphology and the preservation of surface features. Lava flowing down pre-existing wadis acquired an elongated form as they filled the wadis.

Phase 4 overlies the Maradah Formation in some places, but the contact is not always clear due to talus covers, 
but in other places the contact is quite sharp. This phase is represented by dark grey to black vesicular lavas in the upper part with polygonal columnar joints and dense masses in the lower part. This flow is characterized by hilly morphology, pressure ridges and steep distinctly fresher lava scarps. On the satellite image, it has a black colour and features a large number of baltas.

Phase 5 is represented by a well-preserved volcanic form with the freshest of rocks, a vesicular form, and the conspicuous features of the pahohoe structure. The rocks of this phase cover a relatively small area confined to the south-west part of the study area. The excellent preservation and appearance of this phase reflects the younger age of these basaltic flows. On the satellite image the rocks are dark black with dots, representing small baltas of 5 to $20 \mathrm{~m}$ in size.

The drainage pattern developed on the surface of basaltic lava flows shows systematic alteration with the age of the basaltic lava flows, Busrewil and Suwesi [2]. From the Landsat TM image, the drainage frequently decreases as the flows of basaltic rock become progressively younger, as Phase 5 has a less dense drainage pattern.

The Libyan volcanic provinces are spatially related to two structural trends: early Palaeozoic structural elements of a prevalent NNW-SSE direction and by late Palaeozoic to Mesozoic structures with a general ENEWSW trend. These elements represent the regional structures that have played an important role in the geological evolution of Libya, Peregi et al. [5]. Klitzsch [8] suggested that the basalts of Al Haruj al Aswad erupted at the crossing zone of NW trending early Palaeozoic elements (south Al Haruj uplift) and the NE trending late Paleozoic elements (Tibesti-Sirt uplift), and they probably also used some of the upper Cretaceou-Tertiary faults as conduits.

According to Busrewil and Suwesi [2], the major structural trend of the central part of Al Haruj al Aswad volcanic province is controlled by the alignment of spatter cones and the distribution of the more recent lava flows (Phases 5 and 6) along NW-SE and NE-SW directions. Herein, the present area is located to the northeastern part of Al Haruj al Aswad, where the data taken from the investigated area reveal that Phases 4 and 5 are controlled by the NE-SW direction, which is coincident with the pattern of the most distinct structures in the region (Figure 17).

From the direction of flow of the more recent lava (Phases 4, 5, 6) and from the alignment of cones, it is suggested that the situation of the Al Haruj lava field is related to tensional lines, one parallel to the Dur al Qussah fault system and the other running along the prolongation of the Hun graben, Busrewil and Suwesi [2]. These lines probably represent extensional stresses reactived by Proterozoic basement faults in the direction NW-SE, NNW-SSE, NE, NNE and E-W during the late Cenozoic, reflecting the evolution of the Red Sea, EL-Akhal [48]. The rising magma erupted along the fault planes cutting across the lower most resistant areas at the cross points of these tensional lines. The lava field and its associated volcanoes are thus considered to have resulted from a rather continuous release of pressure in the main central feeder. After the activity waned in Al Haruj volcanic province (after the Pleistocene), and the area was cut by a conspicuous rejuvenated NW-SE set of faults, Busrewil and Suwesi [2].

The different rock varieties of the study area are petrographically described in 42 thin sections showing certain petrographic features signifying the different varieties. Mineralogically, most of the studied basaltic rocks contain olivine as an essential mineral together with clinopyroxenes (augite), plagioclase (labradorite to bytownite) feldspars and opaque accessory minerals (probably ilmenite). The recorded olivines are unzoned and either fresh or altered.

Phases 1, 3 and 4 contain a higher modal olivine content and olivine phenocrysts that are larger in size than those from Phases 2 and 5.

The volcanic trends (Phases 4 and 5) are well documented in the area investigated by the continuation of the NE-SW structural trend.

Therefore, the studied rocks can be considered as olivine bearing or olivine basaltic rocks, since they contain a significant amount of modal olivine.

The degree of alteration of the olivines in the studied rocks varies greatly; alteration is manifested around the edges and along the cracks of Phases 3 and 4. On the other hand, highly altered basaltic rocks are found in Phases 1 and 2. The alteration products are either partial or complete to deep reddish iddingsite. Clinopyroxene (augite) is detected in the investigated rocks: it has a maximum extinction angle of $44^{\circ}$ to $51^{\circ}$, and is characterized by a pale brown colour, and is subhedral to anhedral and prismatic in shape. Pleochrism is not observed in most samples.

The rocks from Phase 5 are characterized by low plagioclase modes (labradorite to bytownite) and clinopy- 


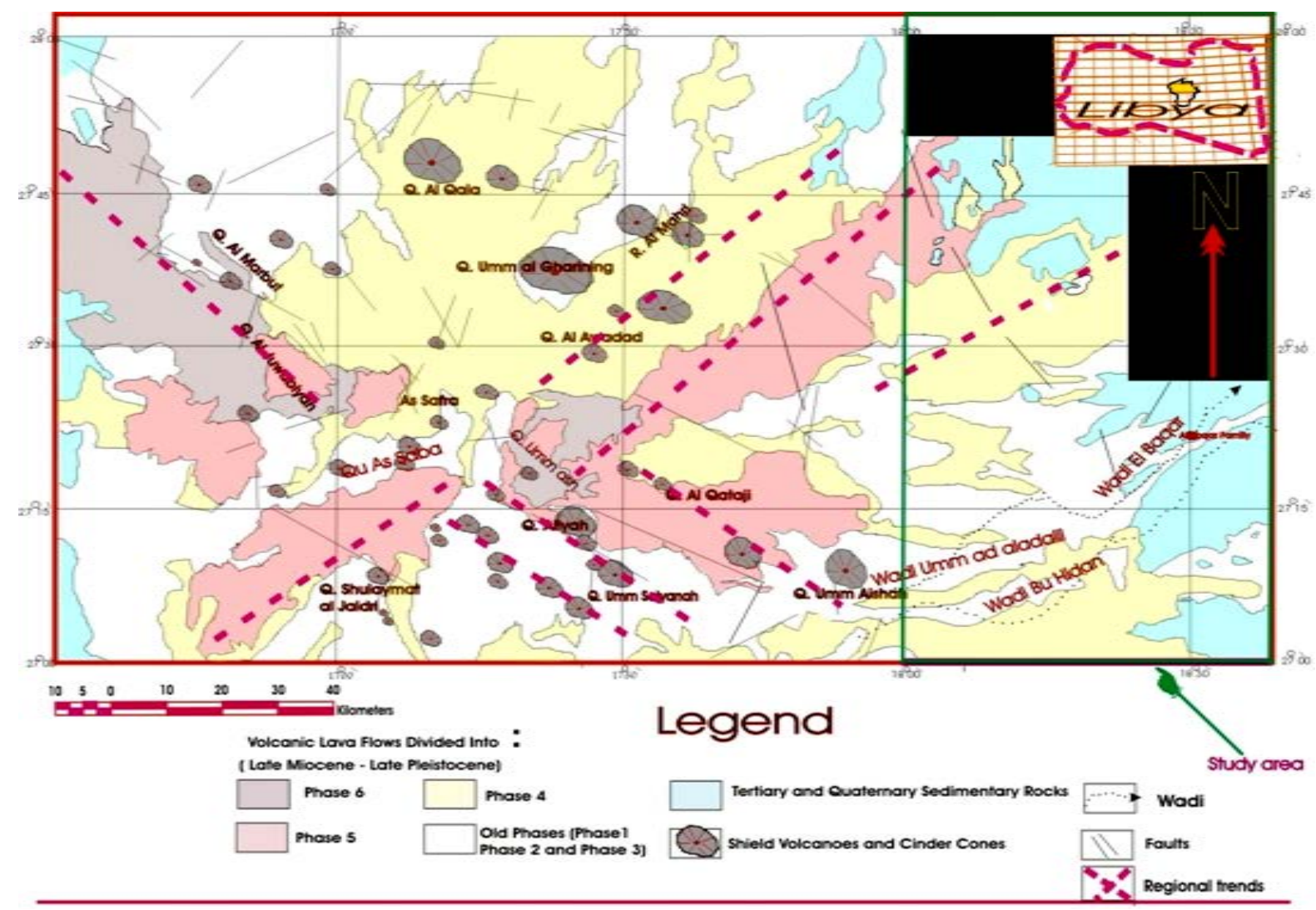

Figure 17. The structural alignment of the central part of al Haruj al Aswad volcanic province (Phases 5 and 6) and a scatter of cones following NE-SW and NW-SE trends, Busrewill and Suwesi [2].

roxene (augite) and high contents of vesicles, amygdales and cryptocrystalline to glassy groundmass and microphenocrysts of olivine, which are fresher when compared to rocks from other phases. Plagioclase laths are the most abundant, representing $37 \%$ to $48 \%$ of the matrix in most of the studied samples. The composition of the plagioclase varied between An 58\% to An 78\% (labradorite to bytownite). They are both pilotaxitically and randomly arranged, and sometimes they exhibit a trachytic arrangement around olivine phenocrysts. Brown glass and cryptocrystals fill the interstices between laths of plagioclase. The rocks exhibit a porphyritic texture with phenocrysts embedded within the groundmass, whereas the groundmass shows intergranular, intersertal, ophitic and subophitic textures. Olivine phenocrysts occur sometimes as clots displaying a glomerporphyritic texture.

The order of crystallization of the minerals in the rocks from the various phases is similar. Phenocrysts of olivine result from a slower cooling beneath the surface in the magma chamber prior to eruption. Therefore, they indicate the presence of enough time to equilibrate with the melt at mantle depth. Upon eruption, the remaining liquid crystallizes to form fine laths and tabular or equant crysts comprising the groundmass and resulting in a dense network of plagioclase microlites and pyroxene granules, with opaque oxides of a small size. Glass may solidify at late stage, filling spaces between minerals and forming an intersertal texture, as in Phase 5.

Inclusions of opaque minerals should have formed at an earlier stage than the plagioclase (labradorite to bytownite) that enveloped them. In the ophitic texture, laths of plagioclase (labradorite to bytownite) enveloped by larger pyroxene (augite) is commonly interpreted as indicating that the clinopyroxene (augite) formed later, Winter, [22]. An anhedral or skeletal form of olivine indicates that their formation is in the later or final stages of rock crystallization, Winter [22]. From petrographical and mineralogical criteria alone, it is evident that the investigated basaltic rocks have more olivine phenocrysts than the pyroxene (augite). The groundmass typically consists of interlocking plagioclase (Labradorite to Bytwonite) laths with stubby prisms of clinopyroxene (augite) granules of olivine and opaque minerals.

This petrography study concluded that the five phases are typical alkaline basalts, which supports the findings 
of the study of Busrewil and Wadsworth [1]. Busrewil and Suwesi [2] suggested that they are a "subalkaline basaltic series which show some similarities except that they are more clearly subophitic with the presence of more opaque minerals". Consequently, the studied mafic volcanic rocks representative of the five phases are probably transitional between alkaline and subalkaline series. But, it must be emphasized that it is necessary to establish their geochemical character to define their petrogenetic affinity within the framework that will establish the magma type. The difference between the various phases is due to the circumstances of magma solidification, not a manifestation of major variances in mineralogical and chemical composition.

The findings of the present study are a positive supplement to previous works such as that by Peregi et al. [5], which suggested that "the petrographic similarity of the mafic rocks that should be noticed, cannot be distinguished frequently in terms of volcanic phases and flow units based on their petrographic characteristics. The difference sometimes may be more significant in samples taken from the surfaces and deeper part of the same lava flow, than between the different volcanic rock phases."

Again, the above study agrees with that by Farahat et al. [14] who stated that "differences in groundmass textures may be related to differential degrees of cooling and crystallization at various levels of the outpouring of magma."

The geochemistry of the basaltic rock under consideration has been revealed through the analysis of 29 samples for their major and trace elements determined quantitatively by Philips (2404) X-ray fluorescence (XRF). Several geochemical parameters and various diagrams were adopted to recognize the chemical characteristics of these rocks, to evaluate their chemistry within the framework of established magma types and to determine the tectonic environment of their phases.

The major elements of the investigated rocks and the content of their trace elements demonstrates that all the phases are similar in chemical composition. In terms of different methods of recent chemical classification based on major, minor and trace elements, the investigated basaltic rocks have a subalkaline to alkaline affinity. If the term "transitional basalts" is accepted, the investigated rocks are best named as "transitional".

The most studied samples are hypersthene-normative. Wilkinson [49] considered hypersthene-normative rocks to be transitional or mild alkaline, and other samples were classified as nepheline-normative or alkaline basalts, and one sample was located at the boundary between olivine tholeiite and quartz tholeiite, which could represent a distinctive feature of tholeiitic basaltic rock.

In the current study, the focus has been on trace elements, which are generated as enriched or depleted and reveal more about the nature of basaltic rocks. Concentrations of compatible trace elements are low in the studied samples, which supports the contention that they are not primary magma. A characteristic feature of the studied volcanic rocks is their relatively constant ratios of certain incompatible trace elements such as $\mathrm{Nb} / \mathrm{Zr}$ and $\mathrm{Rb} / \mathrm{Zr}$. The constancy of incompatible element ratios provides strong evidence that they were generated from a common source. The $\mathrm{Zr} / \mathrm{Nb}$ ratios of the basaltic rocks are less than 10 , which also indicates an OIB source. The studied samples have similar ratios of $\mathrm{Zr} / \mathrm{Nb}$ and $\mathrm{Hf} / \mathrm{Ta}$, so the effect of crustal contamination on the studied basaltic rocks is probably minor.

Based on MORB normalized trace element patterns of representative samples of different volcanic phases, the patterns show fairly close similarities in their shape and concentration range, whereas they are characterized by very slightly different concentrations of some incompatible trace elements within samples of the same phase and also between samples representative of different phases, but those showed a nearly similar pattern in the less incompatible trace elements.

The studied samples of the different phases, which were enriched in all incompatible trace elements over MORB (normalized values greater than one) in proportion to the degree of incompatibility, also showed slightly positive anomalies in $\mathrm{K}, \mathrm{Ba}$ and $\mathrm{Ta}$ and slightly negative anomalies in $\mathrm{Rb}, \mathrm{Nb}$ and $\mathrm{Zr}$. The rocks display similar patterns of peaks and troughs, strongly suggesting that they share a common parent and subsequent processes, Winter [22].

The basaltic samples from the different volcanic phases showed remarkable similarities with each other, but with slight geochemical variations in some incompatible elements. Consequently, the phases cannot be distinguished from one another on the basis of their trace element contents.

Spikes at $\mathrm{Pb}, \mathrm{Ba}$ and $\mathrm{K}$ may reflect an enrichment component, perhaps due to their contamination by continental crust, Winter, [22]. Enriched LIL and the slight alkaline of the studied basaltic rocks could suggest a derivation from an enriched mantle source. The high LIL/HFS pattern and negative $\mathrm{Nb}$ anomaly is a feature of much intracontinental volcanism, suggesting a distinctive feature of the subcontinental lithospheric mantle. The 
studied samples tend to be depleted in Ni and Cr, along with Mg\# (Mg/(Mg + Fet)) (52 - 62), suggesting the fractionation of olivine and spinel prior to eruption.

Despite differences in the major element chemistry, the pronounced similarities in the pattern of trace element between different phases certainly suggest that there is a common source of the mantle peridotite beneath the $\mathrm{Al}$ Haruj volcanic province.

The results of correspondence analysis are explained by the variance on the coordinates (dimensions) of more than $98 \%$ (96.03\% of inertia on dimension 1 and 2.192\% of inertia on dimension 2), which implies that the relation between the average of the different phases is highly significant. The quality of the average of the five phases expresses the value and importance of each phase, so it can clearly be noticed that all phases are of very significant importance.

The basalts of the area representing all phases were formed in an intracontinental (within plate) tectonic setting and they represent alkali to tholeiitic basalts (transitional basalt), most of them having undergone slight to moderate fractionation. The studied basaltic rocks were plotted around the separating line between the two fields (oceanic and continental), Pearce et al., [42]; Figures 5-14(b)). Probably the volcanic activity responsible for these basalts occurred in a zone of initial continental rifting.

The trace element distribution patterns of the studied rocks is similar to OIB, which probably represents basaltic melts from the enriched asthenospheric mantle source, often associated with an uprising mantle plume, Peregi et al. [5].

\section{Comparison of the Tertiary Volcanics from Different Parts of Libya}

Generally, Libya is situated on the Mediterranean foreland of the African shield, which extends over a platform of cratonic basins. The major diastrophic disturbances include the Caledonian and Hercynian disturbances during the Cretaceous, middle Tertiary (Oligocene through to Miocene) and Holocene times, causing uplifts, subsidence, tilting, faulting and intrusions. However, the effects of major diastrophic events were generally broad, and there are very few compressional folds, Goudarzi [50].

Apart from the different ages of the four principal volcanics in Libya, much similarity can be detected among them, particularly in the mechanism and mode of eruption. The common criterion for Tertiary volcanics is that volcanism occurs mainly along well-defined old structural elements. Those structural elements are basically developed from three structural periods characteristic of the middle part of North Africa, Klitzsch [51].

The basement underneath the Tertiary volcanism areas is bound to the overlap of older structural elements forming the boundaries of the major current basins in Libya. The overburden sequence of those areas are of the minimum thickness, and occasionally the Precambrian crystalline rocks are exposed in limited and comparatively small areas such as in Jabal al Hasawinah, west of Jabal Nuqay, and the Tibesti area (Busrewil and Oun [28]; El-Makhrouf [52]; Goudarzi [50]). The volcanic fields of the four principal provinces in Libya are strongly correlated to the current basement elevated areas. Consequently, it seems to be that the attenuation of the crust within specific areas during Paleozoic and Mesozoic times is one of the major contributory factors that facilitated the outpouring in the volcanic provinces in Libya during the Tertiary Period.

The Gharyan volcanic province (GVP) is the smallest of the principal Tertiary volcanic areas in Libya, and it forms a generally continuous sheet in the central part of Jabal Nafusah. The others are Jabal as Swada, Jabal Al Haruj and Jabal Nuqy. These volcanic fields represent the Tertiary-Quaternary outpouring of volcanic activity and define a NNW-SSE linear trend with a decrease in age from NNW to SSE. The GVP lies at the intersection of two major structures, the NNW trending Tripoli-Tibesti uplift formed in Caledonian times, and the E-W trending "Nafusah uplift" formed during the Hercynian orogeny (Anketell and Ghellali [53]; Goudarzi [50]; Mikbel [54]).

The volcanic field of Jabal al Hasawinah is related to the intersection of the early Paleozoic NNW-SSE Tripoli-Tibesti uplift and the Hercynian ENE-WSW Al Qarqaf uplift, (Busrewil and Oun, [28]; Jurak, [55]). Further NE of the Jabal al Hasawinah volcanic field, there is the Al Qarqaf uplift crossing the early Paleozoic NNW-SSE structural element of the Al Haruj uplift; Jabal as Swada is spatially related to this area (Woller and Fediuk, [9]).

The situation of the Al Haruj lava field, contrary to the other volcanic provinces, is related to three structural elements: the early Paleozoic Al Haruj uplift of a NNW-SSE direction, the late Paleozoic to Mesozoic NE fault system that is associated with Tibesti-Sirt uplift, and the prolongation of the Cretaceous-Tertiary Hun graben, Busrewil and Suwesi [2]; Woller and Fediuk [9]). This may account for the greater apparent size of the Al Haruj 
volcanic field compared to the other volcanic provinces, Farahat et al. [14].

Chemical analyses of major, trace and rare earth elements (REE) from $\mathrm{Al}$ Haruj volcanics are presented in Appendix III, El-Shaafi [6], together with analyses of representative rocks from different parts of Libya for comparison purposes (Tables B.1-B.4). The analysed samples from representative rocks from different parts of Libya are depicted on variation diagrams. The consistent patterns of the plotted data suggest that apart from oxidation, there has been little alteration.

The subalkaline basalts from the different volcanics in Libya tend to be relatively evolved with a differentiation index (DI) extending from 29.9 in the most primitive basalts to 39.9 in the most evolved hawaiites, Busrewil and Suwesi, [2]. There are no associated less-evolved rock types exposed that might be representative of the parent magma. All the chemically analysed rocks have relatively high silica and low $\mathrm{TiO}_{2}$ and total alkalis, and although there is some partial overlap with the alkali basalts of the main lava series, they are clearly subalkali. They could be related to other lavas generated at lower pressure and/or higher degrees of partial melting of the parent magma. Fractional crystallization at shallow levels, involving major minerals (ol, cpx, \pm opaques) occurring in the rock, can account for the observed series, Busrewil and Suwesi, [2].

For classifying the rocks on a geochemical basis, the variation of silica and total alkalis is one of the most important discrimination techniques. The IUGS total alkalis (TAS) classification of volcanic rocks of Le Bas et al. [56] is adopted and shown in Figure 18.

The Gharyan volcanics, the present study volcanics and the study made by Busrewil and Suwesi, [2] of the basaltic rocks of the central part of Al Haruj al Aswad, illustrate the suite of subalkaline basalts and alkali basalts, and most of them fall within the basalt field of the TAS diagram. The more differentiated types are simply referred to as hawaiites and mugearites (Figure 19(a)). Twenty-seven samples of the studied basaltic rocks from GVP were chemically analysed, Gafeer [57] for major and trace elements (Tables B.1-B.4, Appendix III). Fresh samples were selected as far as possible and attention was paid to the homogeneity of the samples.

The volcanics in the GVP comprise four petrologic and chronologic phases: the Old Lava Series (OLS); its subordinate, the Young Lava Series (YLS; Early-Eocene to Quaternary); phonolite-trachyite intrusions (Late Eocene-Early Oligocene); and the late volcanic centres (LVC) of the Middle-Late Miocene (see Figure 19(a);

TAS (Le Bas et al. 1986)

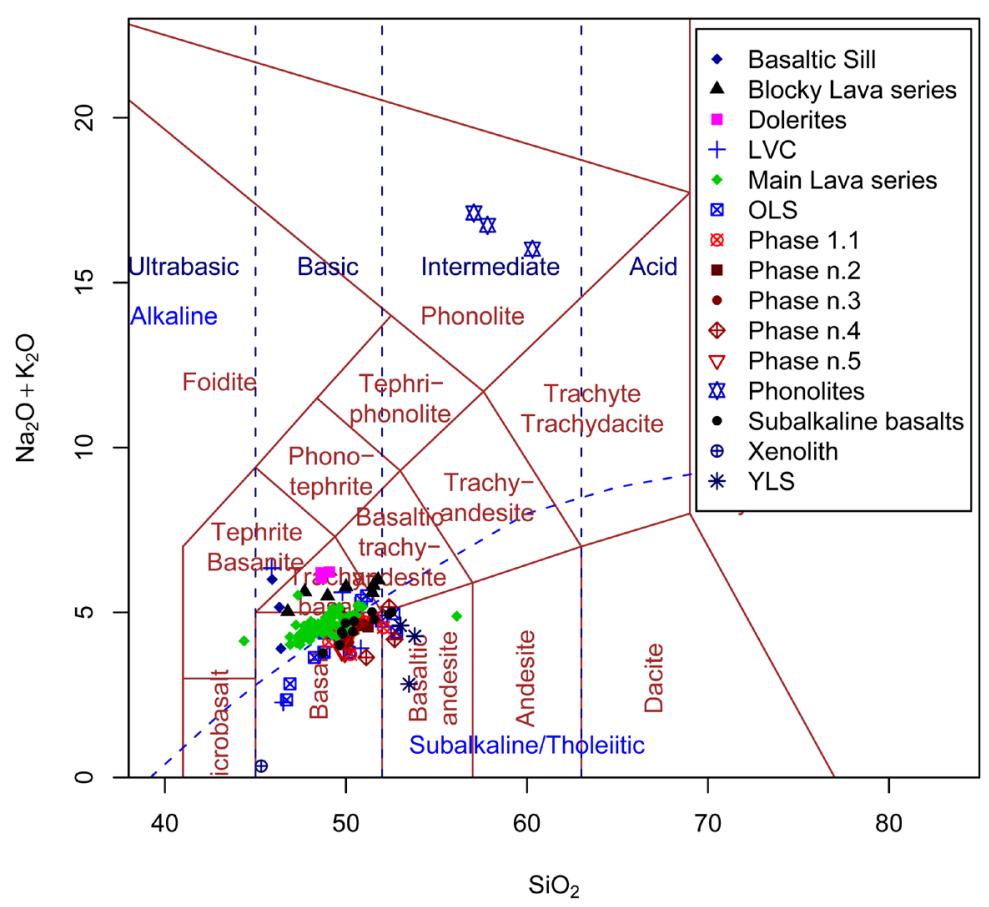

Figure 18. The chemical classification and nomenclature of volcanic rocks studied in the different volcanic provinces in Libya, using the total alkalis versus silica (TAS) diagram of Le Bas et al. [56]. 
Aboazoam et al. [58]; Almond et al. [59]; Busrewil and Wadsworth [1]; Busrewil and Wadsworth [3]; Hegazy [60]; Piccoli [61]).

The Gharyan lava series demonstrates a subalkaline affinity, as shown by its basalt and basaltic andesite presented in Cox et al. ([19]; Figure 19(a), Figure 19(b)), which typically contain normative hypersthene (although three of the analysed samples contain minor amounts of normative nepheline). Generally, the term "transitional" applies to basaltic rocks that exhibit a nepheline-free norm, Le Bas et al. [56].

Wilkinson [49] considered hypersthene-normative rocks to be transitional or mildly alkaline, a characterization which Almond et al. [59] had used to define the suite of the lava series within the Gharyan volcanic province. Phonolites were not observed in Jabal Al Haruj al Aswad by previous investigators or in this study, but the emplacement of phonolites in Jabal Awayant, the Gharyan volcanic province and Jabal Hassawnah, Jurak [55] suggests that a genetic relationship between the basement highs, broad domal uplifts and the emplacement of phonolites exists Busrewil and Suwesi [2].

The divisions of Al Haruj volcanics into plateau lavas, blocky lava, shield volcanoes and doleritic dyke-like masses, based on field appearance have already been established by Busrewil and Suwesi [2]. These data together with chemical observations are used to identify the various rock types present and to assess their petrogenesis. Variations of silica and total alkalis demonstrate a subalkaline affinity trending towards alkaline field (Figure 19(b); in Cox et al. [19]).

Eighty-eight samples of the studied basaltic rocks from Al Haruj al Aswad were chemically analysed by Busrewil and Suwesi [2] for major and trace elements (Tables B.1-B.4, Appendix III). Fresh samples were selected as far as possible and attention was paid to the homogeneity of the samples. Although there is similarity between the lava series of the current study data (Figure 19(c)) to counterparts in the study by Busrewil and Suwesi [2], the above suite shows a wider compositional range as it attains a wider differentiation index (DI) than the present study.

Twenty-nine samples of the studied basaltic rocks from Al Haruj al Aswad were chemically analysed (the current study) for major and trace elements (Tables B.1-B.4, Appendix III; El-Shaafi [6]). Fresh samples were selected as far as possible and attention was paid to the homogeneity of the samples, (Figure 19(c); Cox et al. [19]).

TAS (Cox et al. 1979)

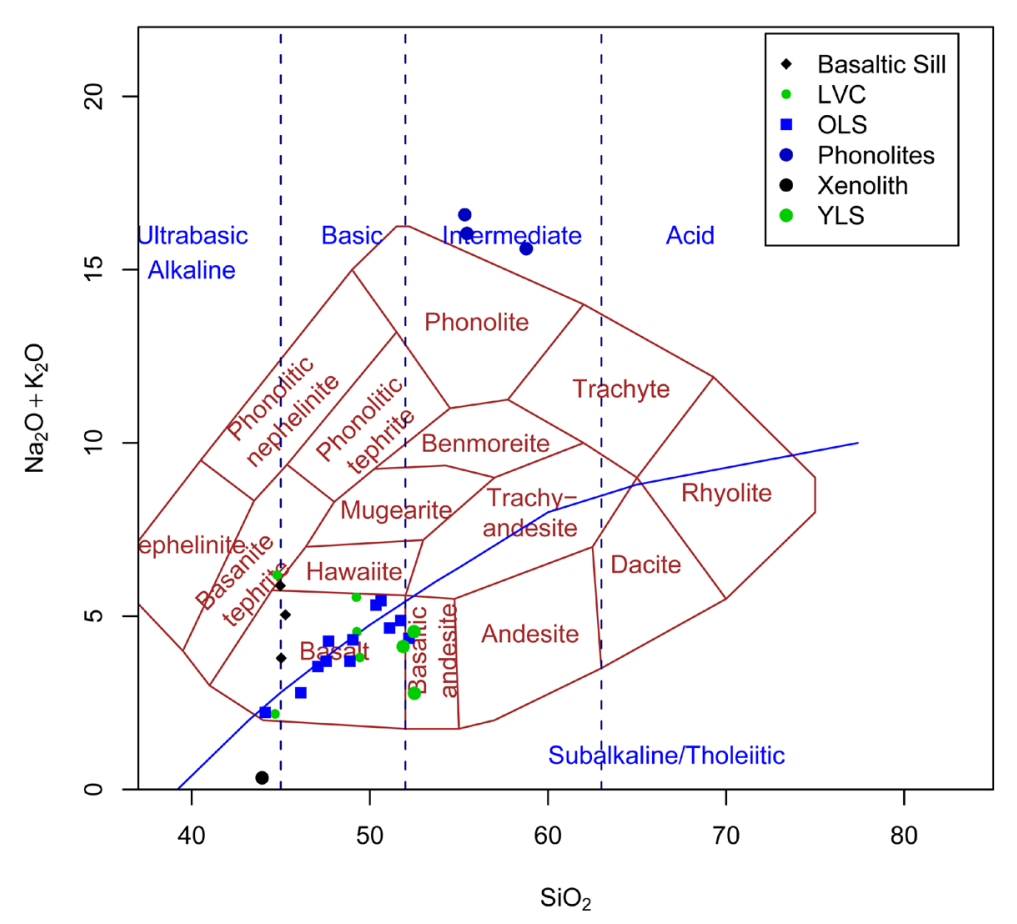

(a) 
TAS (Cox et al. 1979)

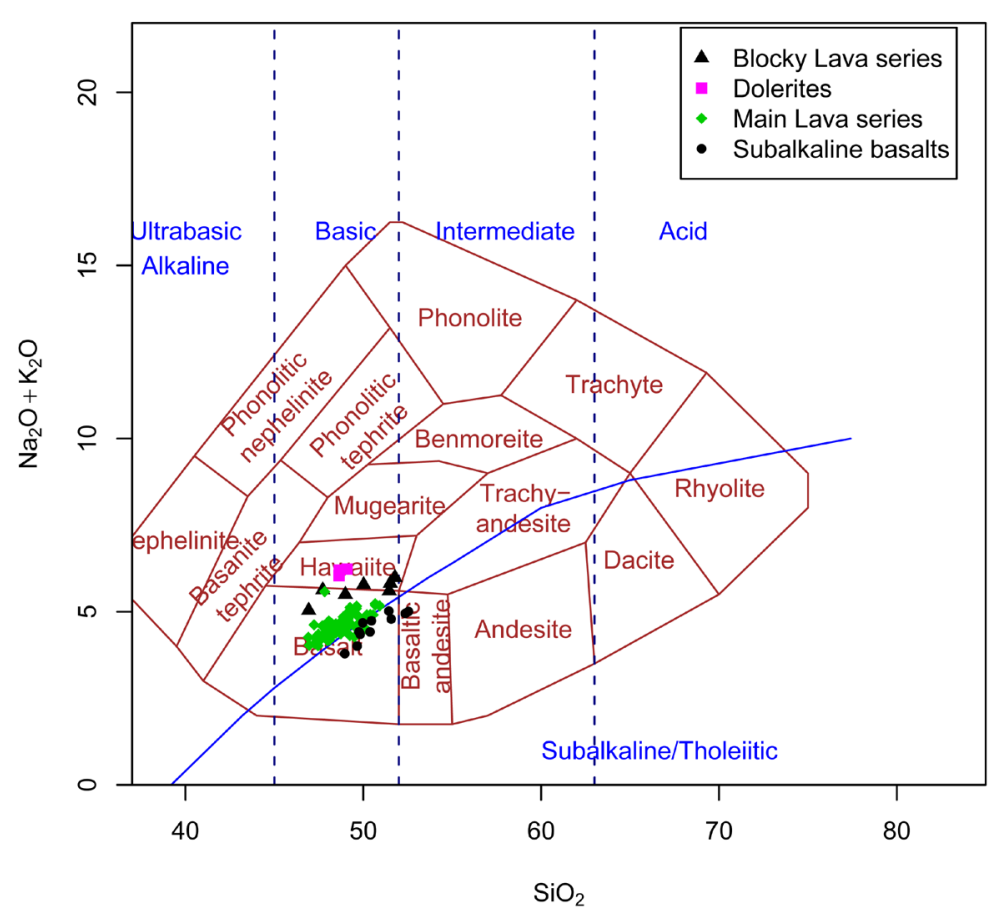

(b)

TAS (Cox et al. 1979)

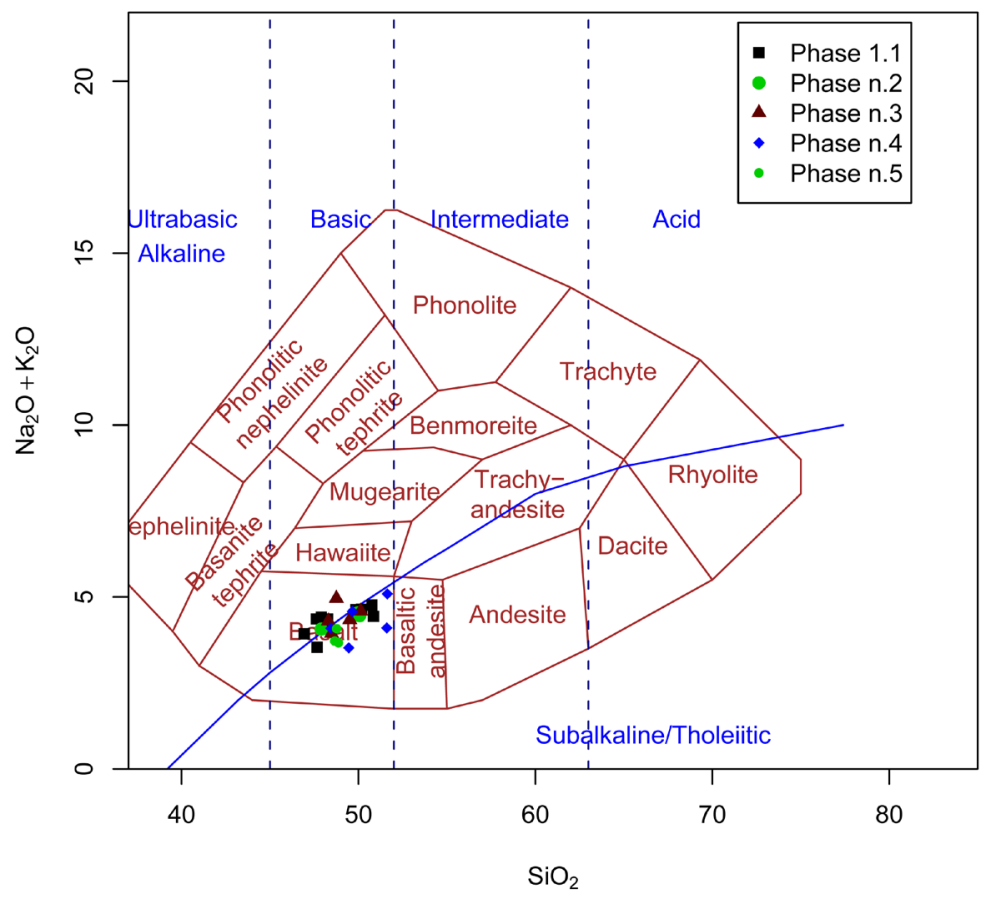

(c)

Figure 19. Alkali versus silica diagrams of Cox et al. [19] showing (a) Plot of total alkalis vs silica for Gharyan province after Gafeer [57]; (b) Plot of total alkalis vs silica from Busrewil and Suwesi [2]; (c) Plot of total alkalis vs silica for the area investigated (current study). 
Based on geochemical data, alkali-olivine basalt and transitional basalt of the study area appear to have been emplaced continentally within plate. The use of immobile HFS elements Th-Hf-Ta is proposed by Wood [47] for basalt plots in the field of "within plate basalts" (Figure 20), whereas the most differentiated members of subalkaline transitional basalts lie in the range of "continental basalts" field on $\mathrm{FeOt}, \mathrm{MgO}, \mathrm{Al}_{2} \mathrm{O}_{3}$, (Figure 21; Pearce et al. [43]), which encompasses both subalkaline basalts and basaltic andesites.

The within plate tendency for the analysed basaltic rocks from various regions are pronounced in the $\mathrm{Zr} / \mathrm{Y}-\mathrm{Zr}$ diagram (Figure 22).

The chemical data for different volcanic provinces are normalized to the primitive mantle (Figure 23) in which the observed $\mathrm{Nb}$ anomaly is a characteristic feature of continental and oceanic alkali basalts. The contamination by crustal rocks is the main factor controlling the behaviour of $\mathrm{K}, \mathrm{Ba}$ and $\mathrm{Rb}$ (Najafi et al., 1981, as cited in Aboazom, [31]).

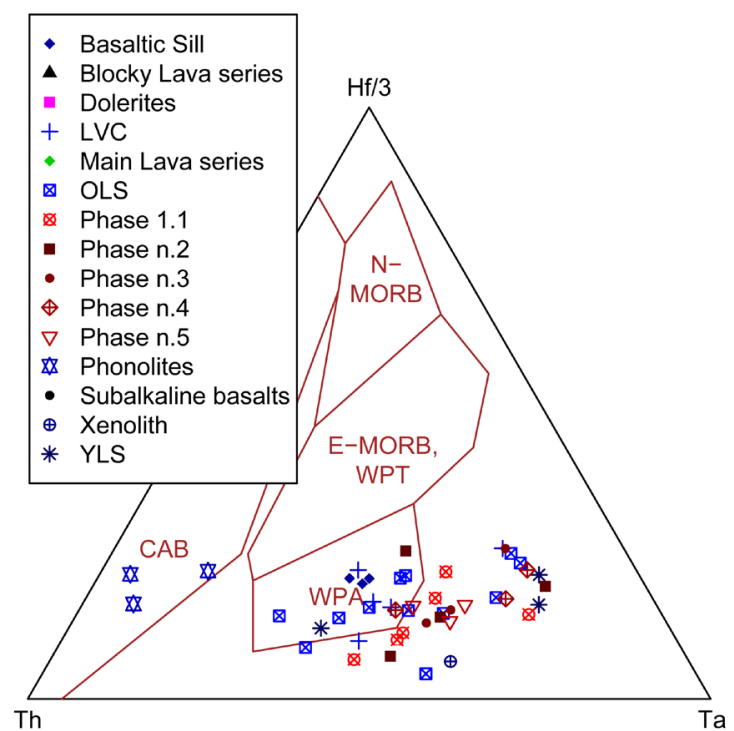

Figure 20. The Th-Hf-Ta discrimination diagram for alkali and tholeiite basalts, after Wood [47].

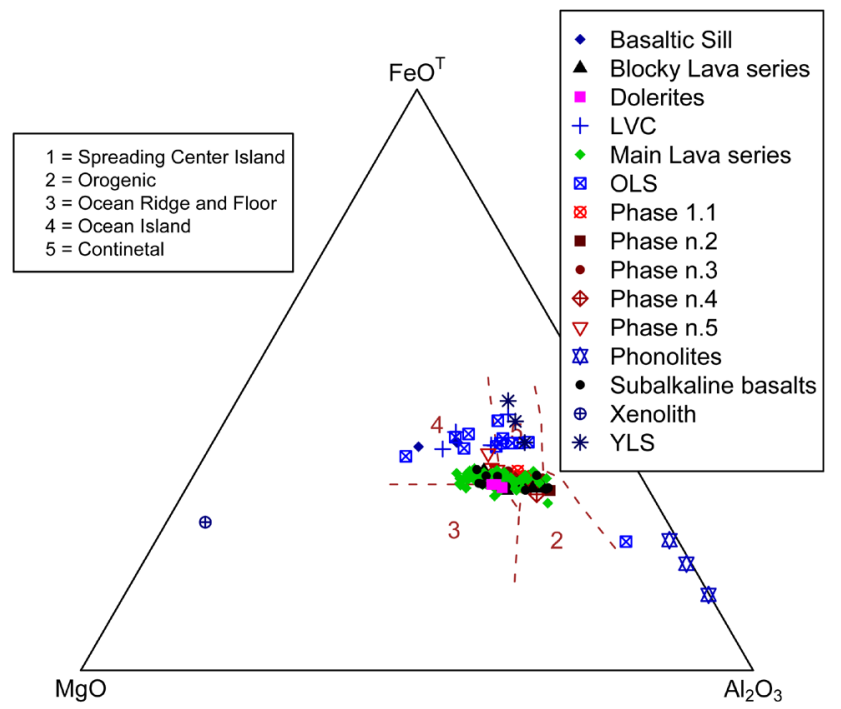

Figure 21. The MgO-FeOt- $\mathrm{Al}_{2} \mathrm{O}_{3}$ diagram, after Pearce et al. [43], showing the discriminant boundaries between tectonic settings based on the compositional range of recent volcanics. The diagram can only used with subalkaline rocks in the silica range of $45 \%-56 \%$. 


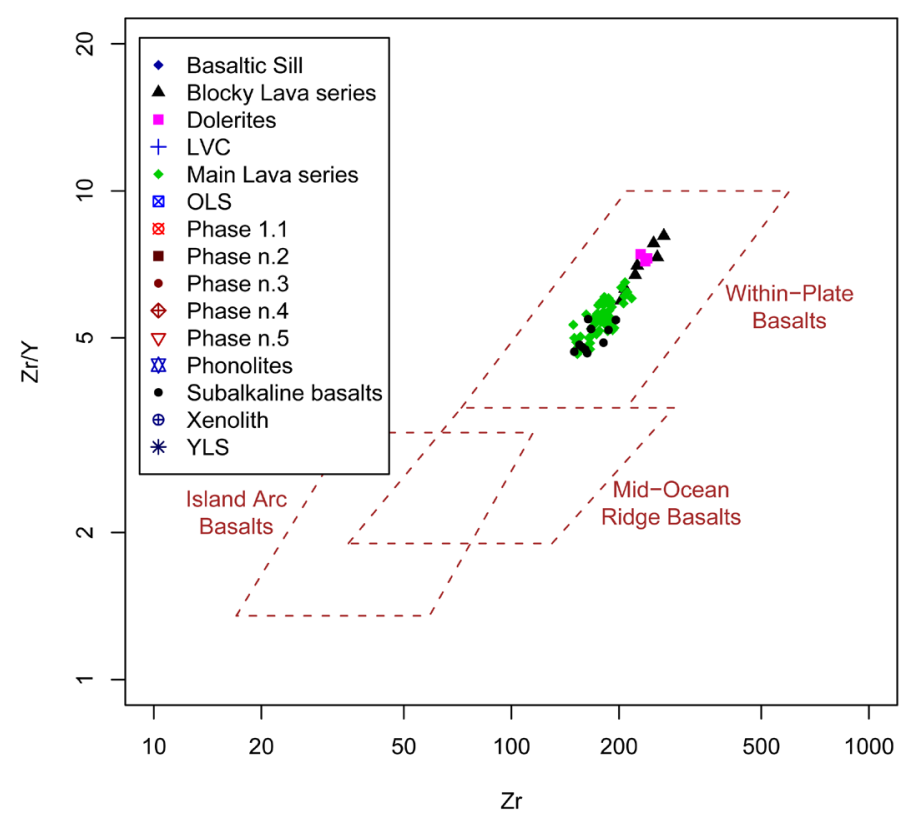

Figure 22. Discrimination diagram for basalts based upon the $\mathrm{Zr}-\mathrm{Zr} / \mathrm{Y}$ variation. The diagram has a logarithmic scale. The analysed samples of Busrewil and Suwesi [2] from the central part of $\mathrm{Al}$ Haruj al Aswad in the field within plate basalts (WPB; after Pearce and Norry, [44]).

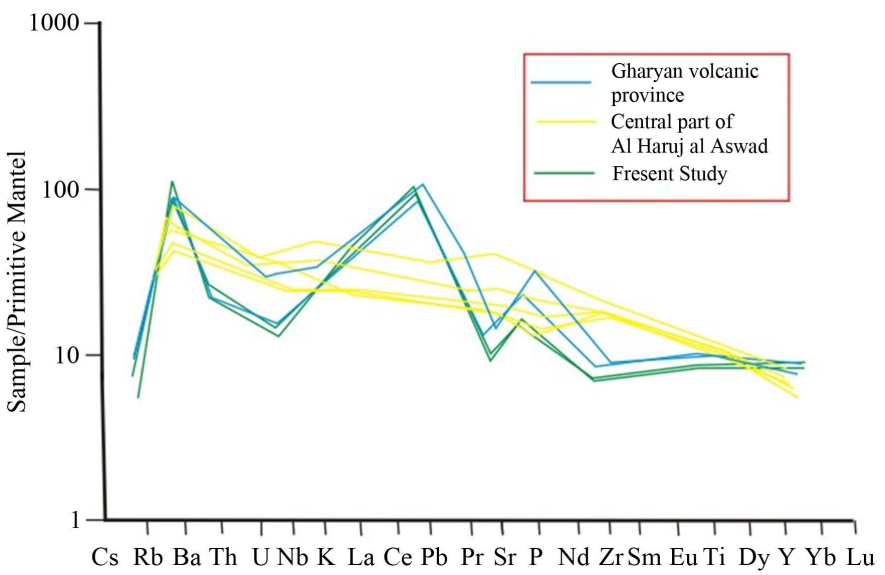

Figure 23. Trace element concentrations of some representative samples from the Gharyan volcanic province by Gaffer [57] and Al Haruj al Aswad by Busrewil and Suwesi [2] and the current study, normalized to the composition of primitive mantle.

The basalts lie within the field of ocean island basalts (Figure 24; Mullen, [62]), which represents basaltic melts from an enriched asthenospheric mantle source. This characteristic has been confirmed for the major outpourings in volcanic provinces in Libya, such as Jabal as Swada, Al Haruj Al-Abiyad (Aboazoam et al., [58]; Hegazzy, [60]; Peregi et al., [5]).

Finally, it is interesting to ask whether the clustering in each and every plot shown in this summary illustrates that the current study represents an evolved portion or a different degree of partial melting from a common igneous source. Further research may give the answer.

\section{Acknowledgements}

The authors would like to express their appreciation to A. El-Arnauti and A. El-Makhrouf for their valuable re- 


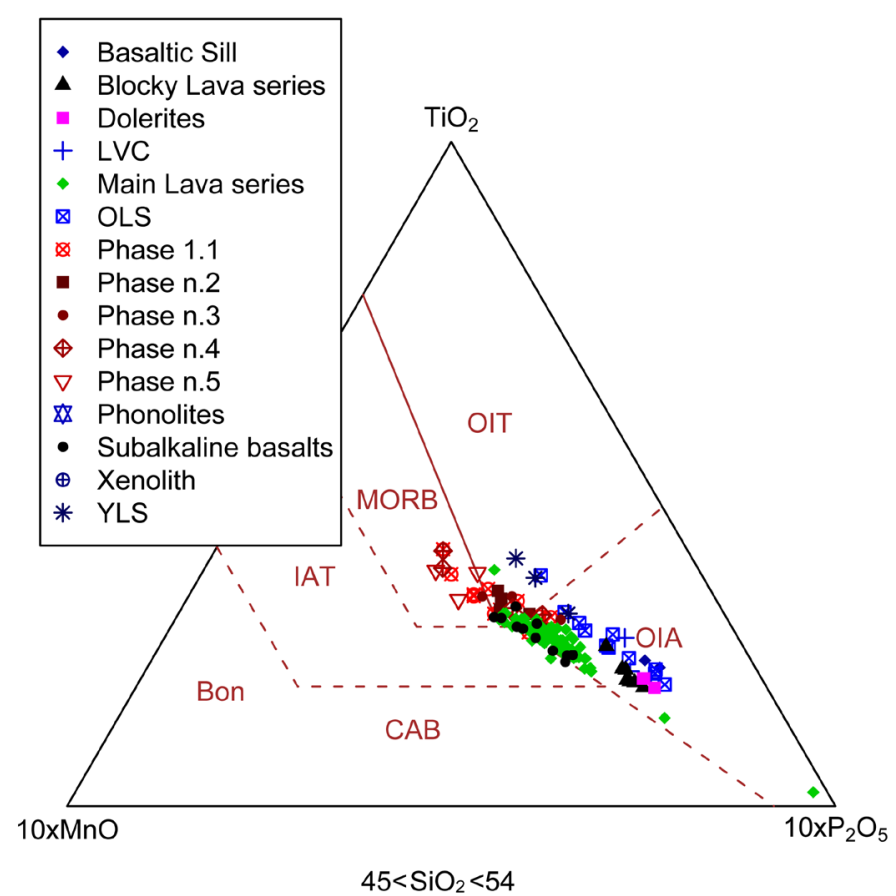

Figure 24. The $\mathrm{MnO}-\mathrm{TiO}_{2}-\mathrm{P}_{2} \mathrm{O}_{5}$ discrimination diagram for basalts and basaltic andesites of $45 \%-54 \% \mathrm{SiO}_{2}$, after Mullen, [62]. The fields are: MORB; OIT-oceanic island tholeiite; OIA-oceanic island alkali basalts; CAB-island arc calc-alkaline basalt; IAT—island arc tholeiite; Bon-boninite.

marks. We feel obliged to express gratitude to M. J. Aburkheis, the former Head of the Popular Committee for the Industrial Research Centre (IRC), and are also indebted to I. Al. Abeid, the Director of the Geological Research and Mining Department and to S. Turki, the Director of the Mapping Department for their encouragement and kind support to the present work while in the field. Thanks and appreciation must go to Rami Gebreel for his valuable help in statistics. Appreciations is expressed to the geological staff of the (IRC): A. Buagrab, A. Garbee, N. Buziad, K. Etrish and Chtronbe from Belgrade University, for their kind assistance during the field work. Thanks are also due to the crew from the IRC mapping project, SE Libya for their help and kind assistance on the camp.

Thanks also extend to M. El-Amawi from the Department of Earth Sciences, University of Benghazi for the coordination and communication with the Central Laboratories of the Geological Survey of Egypt to access the analyses of the samples in question.

\section{References}

[1] Busrewil, M.T. and Wadsworth, W.J. (1980) Preliminary Chemical Data on the Volcanic Rocks of Al Haruj Area, Central Libya. In: Salem, M.J. and Busrewil, M.T., Eds., The Geology of Libya, Vol. 3, Academic Press, London, 1077-1080.

[2] Busrewil, M.T. and Suwesi, S.Kh. (1993) Geological Map of Libya 1:250,000, Sheet. Al Haruj Al Aswad NG $33-4$. Explanatory Booklet. Industrial Research Centre, Tripoli, 95.

[3] Nemeth, K., Suwesi, Kh., Peregi, Z., Gulacsi, Z. and Ujszaszi, J. (2003) Plio/Pleistocene Flood Basalt Related Scoria and Spatter Cones, Rootless Lava Flows and Pit Craters, Al Haruj Al Abiyad, Libya. Geolines, 15, 107-112.

[4] Busrewil, M.T. and Wadsworth, W.J. (1996) Tertiary-Quaternary Alkaline Subalkaline Magmatism in Gharayan Area: Field Aspects and Petrography. PRJ, 8, 13-23.

[5] Peregi, Zs., Less, G.Y., Konrad, Gy., Fodor, L., Gulacsi, Z., Gyalog, L., Turki, S.M., Suwesi, S.Kh., Sherif, Kh. and Dalub, H. (2003) Explanatory Booklet. Geological Map of Libya 1:250,000. Sheet: Al Haruj Al Abyad NG 33-8. Industrial Research Centre, Tripoli, 248.

[6] El-Shaafi Abd El-Salam, S. (2008) Geochemistry and Petrology of Basic Volcanic Rocks of the NE. Part of Jabal AL-Haruj AL-Aswad, Central Libya. Unpublished MSc Thesis, University of Benghazi, Libya. 
[7] Hallett, D. (2002) Petroleum Geology of Libya. Elsevier, Amsterdam.

[8] Klitzsch, E. (2000) The Structural Development of the Murzuq and Kufra Basins: Significance for Oil and Mineral Exploration. In: Sola, M.A. and Worsley, D., Eds., Geological Exploration of the Murzuq Basin, Elsevier, Amsterdam, 143-150. http://dx.doi.org/10.1016/B978-044450611-5/50009-X

[9] Woller, F. and Fediuk, F. (1980) Volcanic Rocks of Gabal as Sawda. In: Salem, M.J. and Busrewil, M.T., Eds., The Geology of Libya, Vol. 3, Academic Press, London, 1081-1093.

[10] Pesce, A. (1966) Uau en Nammus: South Central Libya and Northern Chad. Excursions fừhrer der petroleum Exploration Society of Libya, Tripoli, 47-51.

[11] Ade Hall, F.M., Reynolds, P.H., Dagley, P., Musset, A.G., Hubbard, T.B. and Klitzsch, E. (1974) Geophysical Studies of North African Cenozoic Volcanic Areas Al Haruj Al-Assuad, Libya. Canadian Journal of Earth Science, 11, 9981006. http://dx.doi.org/10.1139/e74-096

[12] Less, Gy., Turki, S.M., Suwesi, S.Kh., Peregi, L.F., Koloszar, L., Kalmar, J., Sherif, Kh., Csaszar, G., Gulasci, Z., Dalum, H. and Al Tajuri, A. (2006) Explanatory Booklet. Geological Map of Libya 1:250,000. Sheet: Waw Al Kabir NG 33-12. Industrial Research Centre, Tripoli, 295.

[13] Klitzsch, E. (1968) Der Basalt Vulkanismus des Djebel Haroudj, Ostfezzan, Libya. Geologische Rundschau, 57, 585609. http://dx.doi.org/10.1007/BF01821263

[14] Farahat, E.S., Abdel Ghani, M.S., Aboazom, A.S. and Asran, A.M.H. (2006) Mineral Chemistry of Al Haruj LowVolcanicity Rift Basalts, Libya: Implications for Petrogenetic and Geotectonic Evolution. Journal of African Earth Sciences, 45, 198-212. http://dx.doi.org/10.1016/j.jafrearsci.2006.02.007

[15] Vesely, J. (1985) Geological Map of Libya 1:250,000 Sheet: Zallah NH 33-16. Explanatory Booklet. Industrial Research Centre, Tripoli, 125.

[16] Woller, F. (1984) Geological Map of Libya 1:250,000, Sheet: Al Fuquaha NG 33-3. Explanatory Booklet. Industrial Research Centre, Tripoli, 123.

[17] Mackenzie, W.S. and Adams, A.E. (1995) A Color Atlas of Rocks and Minerals in Thin Section. Manson, London.

[18] Hatch, F.H., Wells, A.K. and Wells, M.K. (1972) Petrology of the Igneous Rocks, Fifth Impression. Thomas Murby, London.

[19] Cox, K.G., Bell, J.D. and Pankhurst, R.J. (1979) The Interpretation of Igneous Rocks. Allen \& Unwin, London. http://dx.doi.org/10.1007/978-94-017-3373-1

[20] Middlemost, E.A.K. (1980) Iron Oxidation Ratios, Norms and the Classification of Volcanic Rocks. Chemical Geology, 77, 19-26.

[21] Middlemost, E.A.K. (1975) The Basalt Clan. Earth Science Reviews, 11, 337-364. http://dx.doi.org/10.1016/0012-8252(75)90039-2

[22] Winter, J.W. (2001) An Introduction to Igneous and Metamorphic Petrology. Prentice Hall.

[23] Winchester, J.A. and Floyd, P.A. (1976) Geochemical Discrimination of Different Magma Series and Their Differentiation Products Using Immobile Elements. Chemical Geology, 20, 325-343. http://dx.doi.org/10.1016/0009-2541(77)90057-2

[24] Rollinson, H.R. (1993) Using Geochemical Data, Evolution, Presentation, Interpretation. Longman Scientific \& Technical, Oxford/John Wiley, New York, 1-206.

[25] Yoder, H.S. and Tilley, C.E. (1962) Origin of Basalt Magmas: An Experimental Study of Natural and Synthetic Rock Systems. Journal of Petrology, 3, 342-532. http://dx.doi.org/10.1093/petrology/3.3.342

[26] Thompson, R.N. (1984) Dispatches from the Basalts Front. 1. Experiments. Proceedings of the Geologists' Association, 95, 249-262. http://dx.doi.org/10.1016/S0016-7878(84)80011-5

[27] Wilkinson, J.F.G. (1986) Classification and Average Chemical Composition of Common Basalts and Andesites. Journal of Petrology, 27, 181-194. http://dx.doi.org/10.1093/petrology/27.1.31

[28] Busrewil, M.T. and Oun, K.M. (1991) Geochemistry of the Tertiary Alkaline Rocks of Jabal al Hasawnah, West Libya. In: Salem, M.J., Busrewil, M.T. and Ben Ashour, A.M., Eds., Geology of Libya, Vol. 7, Elsevier, Amsterdam, 25872598.

[29] Baker, B.H. (1987) Outline of the Petrology of the Kenya Rift Alkaline Province. In: Fitton, J.G. and Upton, B.J., Eds., Alkaline Igneous Rocks, Geological Society, Special Publications, London, 293-311.

[30] Pearce, J.A. (1983) Lithosphere in Magma Genesis at Active Continental Margins. In: Hawesworth, C.J. and Norry, M.J., Eds., Continental Basalts and Mantle Xenoliths, Shiva.

[31] Aboazom, A.S. (2005) Petrogenesis of Phase 6 of Al Haruj Al Aswad Volcanic Province, Central Libya. Sebha University Journal, 4, 96-113. 
[32] Fitton, J.G., James, D., Kempton, P.D., Ormerod, D.S. and Leeman, W.P. (1988) The Role of the Lithospheric Mantle in the Generation of Late Cenozoic Basic Magmas in the Western United States. J. Petrol. Special Lithosphere Issue, 331-349.

[33] Hawkesworth, C.J., Hempton, P.D., Ellam, R.M. and Calsteren, P. (1990) Continental Mantle Lithosphere and Shallow Level Enrichment Processes in the Earth’s Mantle. Earth and Planetary Science Letters, 96, 256-268. http://dx.doi.org/10.1016/0012-821X(90)90006-J

[34] Hopper, P.R., Bailey, D.G. and Hawkeworth, C.J. (1993) Isotopic and Geochemical Constraints on the Origin and Evolution of the Columbia River Basalt. Journal of Petrology, 34, 1203-1246. http://dx.doi.org/10.1093/petrology/34.6.1203

[35] Longmuir, C.H., Beneder, J.F., Bence, A.E., Hanson, G.N. and Taylor, S.R. (1977) Petrogenesis of Basalts from the Famous Area: Mid-Oceanic Ridge. Earth and Planetary Science Letters, 36, 133-156. http://dx.doi.org/10.1016/0012-821X(77)90194-7

[36] Kay, R.W. and Hubbard, N.J. (1978) Trace Elements in Ocean Ridge Basalts. Earth and Planetary Science Letters, 38, 95-116. http://dx.doi.org/10.1016/0012-821X(78)90128-0

[37] Sun, S.S. (1980) Lead Isotopic Study Volcanic Rocks from Mid-Ocean Ridges, Ocean Islands and Islands Arcs. Philosophical Transactions of the Royal Society A, 297, 409-445. http://dx.doi.org/10.1098/rsta.1980.0224

[38] Pearce, J.A. (1976) Statistical Analysis of Major Element Patterns in Basalts. Journal of Petrology, 17, 15-43. http://dx.doi.org/10.1093/petrology/17.1.15

[39] Pearce, J.A. (1980) Geochemical Evidence for the Genesis and Eruptive Settings of Lavas from Tethyan Ophiolites. Proceedings of the International Ophiolite Symposium, Cyprus 1979, Institute of Mining and Metallurgy, 261-272.

[40] Pearce, J.A. and Cann, J.R. (1971) Ophiolite Origin Investigated by Discriminants Analysis Using Ti, Zr and Y. Earth and Planetary Science Letters, 12, 339-349. http://dx.doi.org/10.1016/0012-821X(71)90220-2

[41] Pearce, J.A. and Cann, J.R. (1973) Tectonic Setting of Basic Volcanic Rocks Determined Using Trace Element Analyses. Earth and Planetary Science Letters, 19, 290-300. http://dx.doi.org/10.1016/0012-821X(73)90129-5

[42] Pearce, T.H., Gorman, B.E. and Birkett, T.C. (1975) The $\mathrm{TiO}_{2}-\mathrm{K}_{2} \mathrm{O}-\mathrm{P}_{2} \mathrm{O}_{5}$ Diagram: A Method of Discriminating between Oceanic and Non-Oceanic Basalts. Earth and Planetary Science Letters, 24, 419-426. http://dx.doi.org/10.1016/0012-821X(75)90149-1

[43] Pearce, T.H., Gorman, B.E. and Birkett, T.C. (1977) The Relation between Major Element Chemistry and Tectonic Environment of Basic and Intermediate Volcanic Rocks. Earth and Planetary Science Letters, 36, 121-132. http://dx.doi.org/10.1016/0012-821X(77)90193-5

[44] Pearce, J.A. and Norry, M.J. (1979) Petrogenetic Implications of Ti, Zr, Y and Nb Variations in Volcanic Rocks. Contributions to Mineralogy and Petrology, 69, 33-47. http://dx.doi.org/10.1007/BF00375192

[45] Wood, D.A., Tarney, J., Varet, J., Saunders, A.D., Bougault, H., Joron, J.L., Treuil, M. and Cann, J.R. (1979) Geochemistry of Basalts Drilled in the North Atlantic by IPOD Leg 49: Implications of Mantle Heterogeneity. Earth and Planetary Science Letters, 42, 77-79. http://dx.doi.org/10.1016/0012-821X(79)90192-4

[46] Floyd, P.A. and Winchester, J.A. (1975) Magma Type and Tectonic Setting Discrimination Using Immobile Elements. Earth and Planetary Science Letters, 27, 211-218. http://dx.doi.org/10.1016/0012-821X(75)90031-X

[47] Wood, D.A. (1980) The Application of a Th-Hf-Ta Diagram to Problems of Tectonomagmatic Classification and to Establishing the Nature of Crustal Contamination of Basaltic Lavas of the British Tertiary Volcanic Province. Earth and Planetary Science Letters, 50, 11-30. http://dx.doi.org/10.1016/0012-821X(80)90116-8

[48] El-Akhal, H. (2004) Contribution to the Petrology, Geochemistry and Tectonic Setting of the Basalt Flows of the Umm-Qais Plateau, North Jordan.

[49] Wilkison, J.F.G. (1986) Classification and Average Chemical Composition of Common Basalts and Andesites. Journal of Petrology, 27, 181-194.

[50] Goudarzi, G.H. (1980) Structure: Libya. In: Salem, M.J. and Busrewil, M.T., Eds., The Geology of Libya, Vol. 3, Academic Press, London, 879-892.

[51] Klitzsch, E. (1971) The Structural Development of Parts of North Africa since Cambrian Times. In: Gray, C., Ed., First Symposium on the Geology of Libya, Faculty of Science, University of Libya, Tripoli, 253-262.

[52] El-Makhrouf, A.A. (1988) Tectonic Interpretation of Jabal Eghei Area and Its Regional Application to Tibesti Orogenic Belt, South Central Libya. Journal of African Earth Science, 7, 945-967. http://dx.doi.org/10.1016/0899-5362(88)90009-7

[53] Anketell, J.M. and Ghellali, S.M. (1991) A Palaeogeologic Map of the Pre-Tertiary Surface in the Region of the Jifarah Plain and Its Implication to the Structural History of Northern Libya. In: Salem, M.J., Busrewil, M.T. and Ben Ashour, A.M., Eds., Geology of Libya, Vol. 7, Elsevier, Amsterdam, 2681-2687. 
[54] Mikbel, S.R. (1977) Basement Configuration and Structure of West Libya. Libyan Journal of Science, 7A, $19-33$.

[55] Jurak, L. (1978) Geologic Map of Libya 1:250,000, Sheet: Jabal Al Hasawanah NH 33-14. Explanatory Booklet. Industrial Research Centre, Tripoli, 74-100.

[56] Le Bas, M.J., LeMaitre, R.W., Strecheisen, A.L. and Zanettin, B. (1986) A Chemical Classification of Volcanic Rocks Based on the Total Alkali-Silica Diagram. Journal of Petrology, 27, 745-750. http://dx.doi.org/10.1093/petrology/27.3.745

[57] Gafeer, A.S. (2007) The Petrology and Geochemistry of Gharyan Volcanic Province, NW Libya. Unpublished MSc Thesis, The University of Garyounis, Benghazi.

[58] Aboazom, A.S., Asran, A.S.H., Abdel Ghani, M.S. and Farhat, E.S. (2006) Geologic and Geochemical Constraints on the Origin of Some Tertiary Alkaline Rift Volcanics, Gharyan Area, Northwestern Libya. Assiuit University Journal of Geology, 35, 25-47.

[59] Almond, D.C., Busrewil, M.T. and Wadsworth, W.J. (1974) The Gharian Tertiary Volcanic Province of Tripolatania, Libya. Geological Journal, 9, 17-28. http://dx.doi.org/10.1002/gj.3350090102

[60] Hegazzy, A.H. (1999) Tertiary Volcanics in Libya: Evidence for the Direction and Rate of the African Plate Motion. Proceedings of the Fourth International Conference on Geochemistry, Alexandria University, Egypt, 401-419.

[61] Piccoli, C. (1971) Outlines of Volcanism in Northern Tripolitania. In: Gray, C., Ed., Symposium on the Geology of Libya, Fac. Sci., Univ. Padova.

[62] Mullen, E.D. (1983) $\mathrm{MnO} / \mathrm{TiO}_{2} / \mathrm{P}_{2} \mathrm{O}_{5}$ : A Minor Element Discriminant for Basaltic Rocks of Oceanic Environments and Its Implications for Petrogenesis. Earth and Planetary Science Letters, 62, 53-62. http://dx.doi.org/10.1016/0012-821X(83)90070-5 
Scientific Research Publishing (SCIRP) is one of the largest Open Access journal publishers. It is currently publishing more than 200 open access, online, peer-reviewed journals covering a wide range of academic disciplines. SCIRP serves the worldwide academic communities and contributes to the progress and application of science with its publication.

Other selected journals from SCIRP are listed as below. Submit your manuscript to us via either submit@scirp.org or Online Submission Portal.
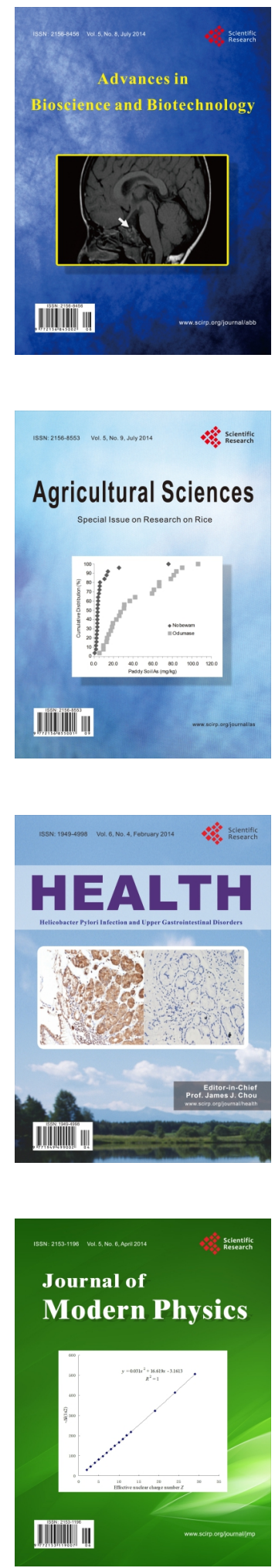
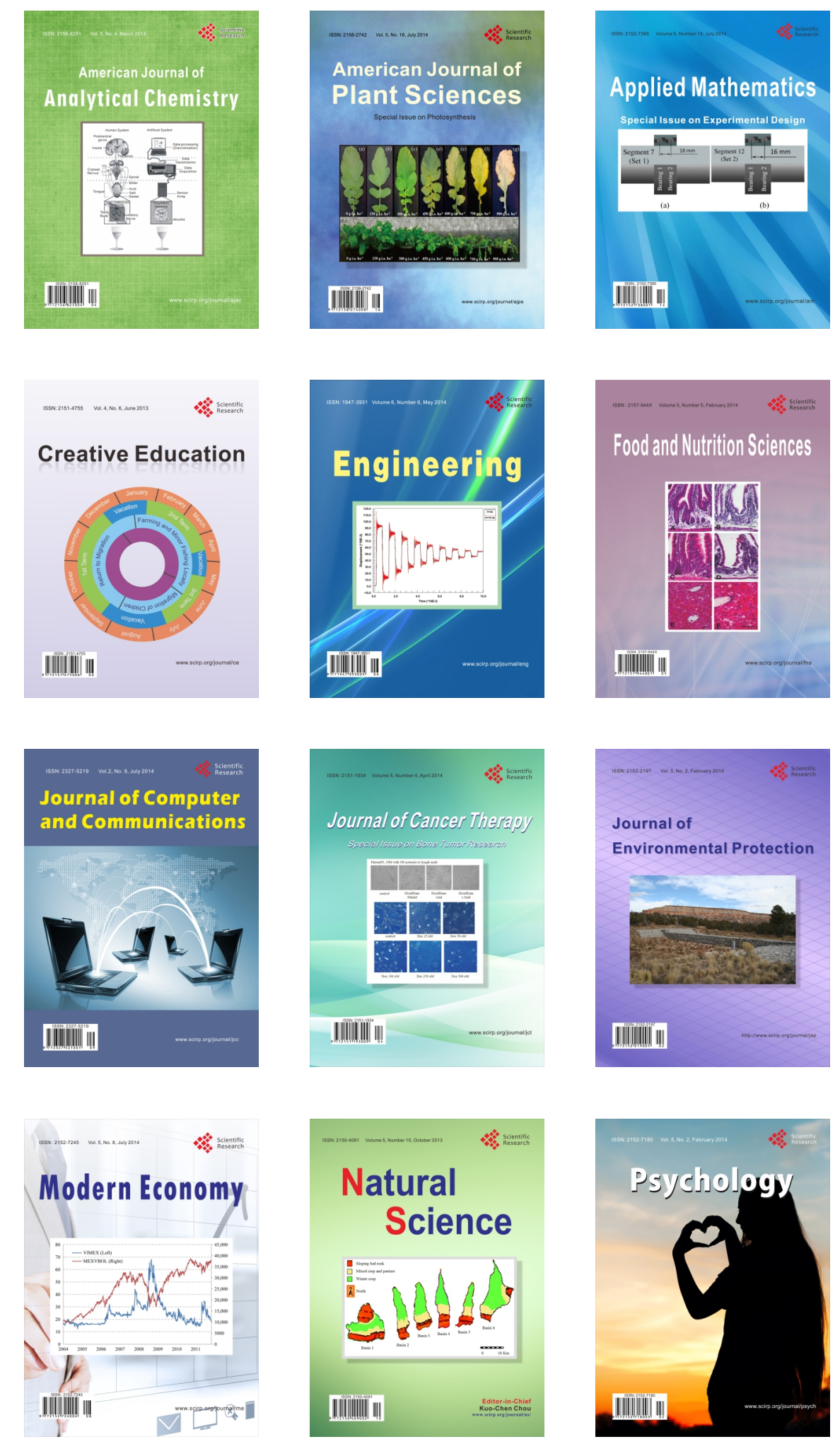